

\section{DISCLAIMER}

This report was prepared as an account of work sponsored by an agency of the United States Government. Neither the United States Government nor any agency Thereof, nor any of their employees, makes any warranty, express or implied, or assumes any legal liability or responsibility for the accuracy, completeness, or usefulness of any information, apparatus, product, or process disclosed, or represents that its use would not infringe privately owned rights. Reference herein to any specific commercial product, process, or service by trade name, trademark, manufacturer, or otherwise does not necessarily constitute or imply its endorsement, recommendation, or favoring by the United States Government or any agency thereof. The views and opinions of authors expressed herein do not necessarily state or reflect those of the United States Government or any agency thereof. 


\section{DISCLAIMER}

Portions of this document may be illegible in electronic image products. Images are produced from the best available original document. 
Frinted in the United States of America. Available from Clearinghouse for Federal Scientific and Technical Information, National Bureau of Standards,

U.S. Department of Commerce, Springfield, Virginia 22151

Price: Printed Copy $\$ 3.00$; Microfiche $\$ 0.65$

\section{LEGAL NOTICE}

This report was prepared as an account of Government sponsored work. Neither the United States, nor the Commission, nor any person acting on behalf of the Commission:

A. Makes any warranty or representation, expressed or implied, with respect to the accuracy, completeness, or usefulness of the information contained in this report, or that the use of any information, apparatus, method, or process disclosed in this report may not infringe privately owned rights; or

B. Assumes any liabilities with respect to the use of, or for damages resulting from the use of any information, apparatus, method, or process disclosed in this report.

As used in the above, "person acting on behalf of the Commission" includes any employee or contractor of the Commission, or employee of such contractor, to the extent that such employee or contractor of the Commission, or employee of such contractor prepares, disseminates, or provides access to, any information pursuant to his employment or contract with the Commission, or his employment with such contractor. 


\title{
A NUMERICAL INVESTIGATION OF A \\ CONFINED VORTEX PROBLEM
}

\author{
Robin E. Textor
}

\author{
Computing Technology Center
}

Submitted as a thesis to the Graduate Council of the University of Tennessee in partial fulfillment of the requirements for the degree Master of Science.

\author{
UNION CARBIDE CORPORATION \\ NUCLEAR DIVISION \\ Computing Technology Center \\ Oak Ridge, Tennessee
}

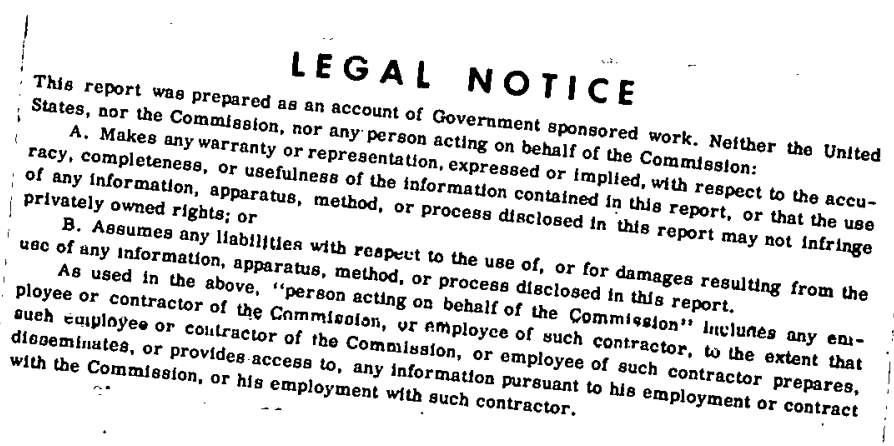




\begin{abstract}
In terms of cylindrical coordinates; the Navier-Stokes equations and the equation of continuity are derived for axisymetric, incompressible flows wherein the pressure is the only important external force. A problem is described in which a cylinder containing fluid and a coaxial tube are in rotation with one of the cylinder end walls at a constant angular velocity, the other cylinder end wall remaining stationary. A successive overrelaxation iterative procedure is described whereby the system of partial differential equations involving the classical stream function and vorticity are solved at steady-state. Some observations of the solutions thus obtained are made for tangential Reynolds numbers from 1 to 2000. Four other methods are discussed by which solutions of the problem either have been or may be attempted.
\end{abstract}


These studies were performed at the Computing Technology Center operated by the Union Carbide Corporation, Nuclear Division for the U. S. Atomic Energy Commission. The author owes a special debt of gratitude to Dr. D. W. Lick, whose courses provided the inspiration for work in the numerical solution of partial differential equations and whose insight, advice and encouragement have proved to be invaluable in this effort. Also, the author would like to express his appreciation to Dr. G. J. Farris, who provided valuable counsel with regard to the physical aspects of the problem and who oversaw the calculations. Finally the author would like to thank Miss Marsha Willien for typing this thesis and Mr. R. E. Graham for preparing the figures. 
I. INTRODUCTION. . . . . . . . . . . . . . . . . . 1

II. DERIVATION OF THE GOVERNING EQUATIONS . . . . . . . . 3

III. CONFINED VORTEX PROBLEM . . . . . . . . . . . . . 23

IV. THE ClASSICAL APPROACH. . . . . . . . . . . . 28

V. OTHER APPROACHES. . . . . . . . . . . . . . 36

LIST OF REFERENCES. . . . . . . . . . . . . . . . 51 


\section{LIST OF FIGURES}

FIGIJRES

PAGE

1. Buildup to Steady Laminar Velocity Profile for

Fluid Contained Between Two Plates. . . . . . . . 4

2. Volume Element in Cylindrical Coordinates . . . . . . . 7

3. Directions in which the R-Component of Momentum is

Transported Through a Volume Element. . . . . . . . 10

4. Structure of the Grid Network . . . . . . . . . . . 29

5. Relation of Field Variables to Grid Structure . . . . . 45 
CHAPTER I

\section{INTRODUCTION}

In recent years a great deal of effort has been made to determine velocity distributions in a variety of fluid flow problems by numerical techniques using digital computers. Such problems are usually represented by a system of partial differential equations, at least some of which are nonlinear, with appropriate restrictions on the solution at the boundary of the region of interest. This thesis will discuss an attempt to find the velocity distributions in a fluid which is essentially flowing in a potential vortex (a rotating flow in which the tangential velocity varies inversely with radial distance) adjacent to a stationary surfact.

Chapter II contains the basic concepts of fluid flow and the derivation of two systems of partial differential equations which describe a class of fluid flow problems. The derivations are performed in cylindrical coordinates but are otherwise carried out in the manner of Bird, Stewart and Lightfoot [1].

In Chapter III the confined vortex with which this thesis is concerned is'described and boundary conditions are provided for the two systems of equations derived in Chapter II.

For finite difference approximations of the classical stream function-vorticity partial differential equations, an iterative method of solution is described in Chapter IV. A criterion for the convergence of this iterative procedure is provided and some observations of the solutions thus obtained are made. 
Chapter V contains a discussion of four other methods for seeking solutions of this confined vortex problem. Two of these methods have been tried with little or no success and the other two methods have not been tried but seem promising. 
In this chapter we seek to exhibit the basic concepts involved in the flow of fluids and, through the use of the principles of conservation of mass and momentum, to derive the partial differential equations which describe a class of fluid flow problems.

Consider a fluid contained between two large parallel piates of area $A$ which are separated by a small distance $Z$, as in Figure 1 . Imagine that the system is initially at rest and that at time $t=0$ we set the lower plate in motion in the $r$-direction with a constant velocity $V$. As time proceeds the fluid gains momentum, and finally the steady-state velocity profile shown in Figure 1 is established. When this steady-state motion has been attained, a constant force $F$ is required to maintain the motion of the lower plate. Provided that the flow is laminar, this force may be expressed as

$$
\frac{F}{A}=\mu \frac{V}{Z}
$$

This equation states that the force per unit area is proportional to the velocity decrease in the distance $z$. The constant of proportionality $\mu$ is called the "viscosity" of the fluid and characterizes the resistance of simple fluids to motion.

The shear stress exerted in the r-direction on a fluid surface at $z=z_{0}$ by the fluid in the region $z<z_{0}$ is designated by $\tau_{z r}$, and the $r$-component of the fluid velocity vector is designated by $u$. 


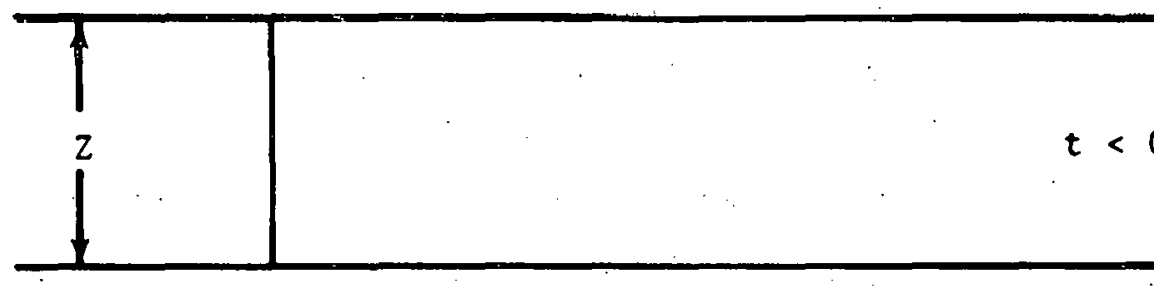

Fluid at rest

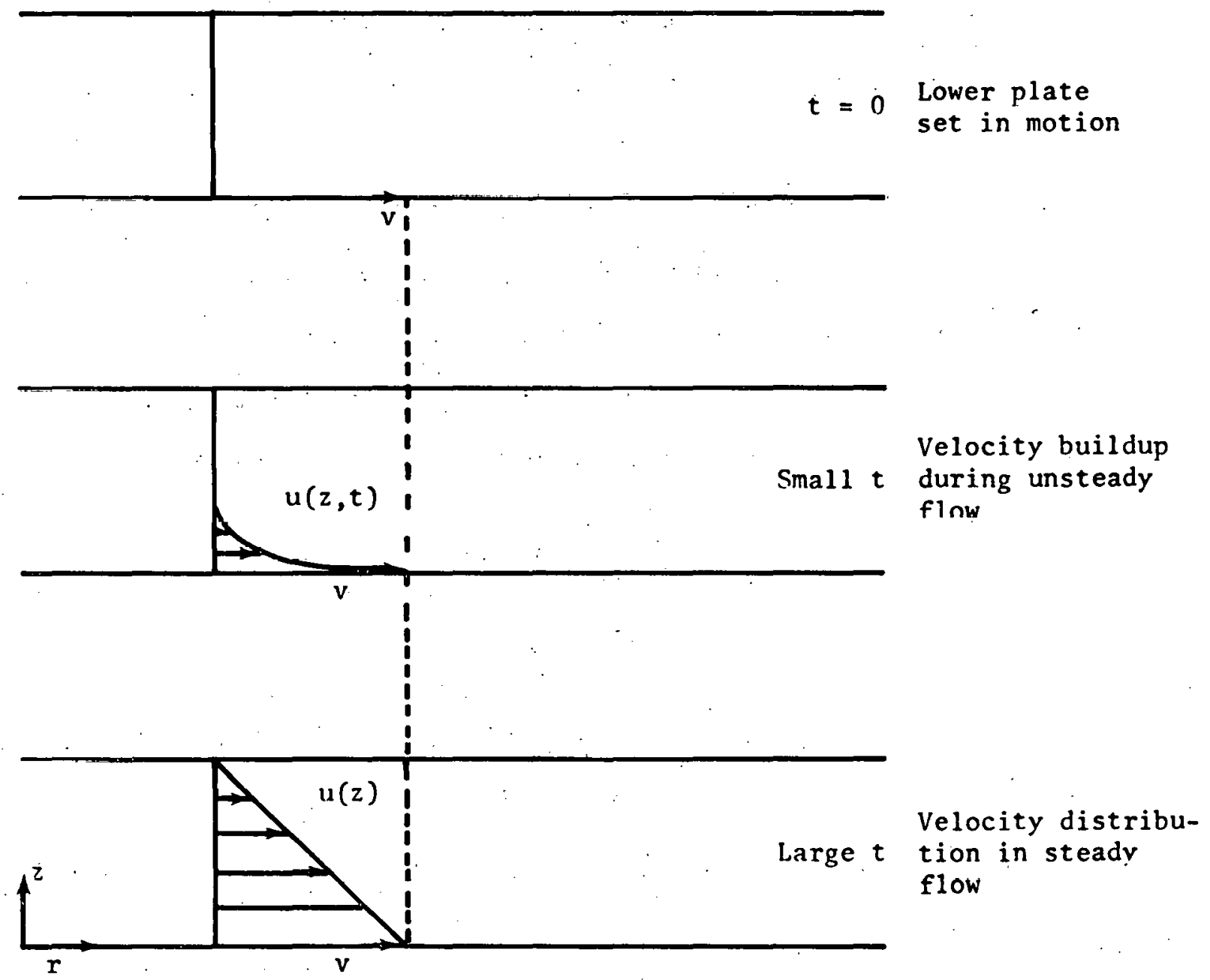

Figure 1. Buildup to Steady Laminar Velocity Profile for Fluid Contained Between Two Plates. 
Note that this is not the rate of change of the velocity $\vec{v}$ in the $r-$ direction designated by $\partial \vec{v} / \partial r$. Now, Equation (2.1) may be rewritten as

$$
\tau_{z r}=-\mu \frac{\partial u}{\partial z}
$$

This equation is known as Newton's law of viscosity and may be stated. as, "The shear force per unit area is proportional to the negative of the local velocity gradient." All gases and most simple liquids are described by Equation (2.2) and are called "Newtonian" fluids.

Another interpretation of Equation (2.2) may be obtained by considering a neighborhood of the moving surface. The fluid in this neighborhood acquires a certain amount of $r$-momentum. The fluid, in turn, imparts some of its momentum to the adjacent "layer" of fluid causing it to remain in motion in the $r$-direction. Thus $r$-momentum is transmitted through the fluid in the $z$-direction. Now $\tau_{z r}$ may be interpreted as the viscous flux of $r$-momentum in the $z$-direction. Note that momentum tends to go in the direction of decreasing velocity and the velocity gradient may be thought of as a "driving force" for momentum transport.

The mass density or mass per unit volume of the fluid is designated by $\rho$ and, since we will later find use for a symbol which represents the viscosity divided by the mass density, we define the kinematic viscosity v. by

$$
v=\mu / \rho
$$


Consider a stationary volume element in cylindrical coordinates, as shown in Figure 2, through which fluid is flowing. Conservation of mass may be stated as the mass balance

$$
\left\{\begin{array}{l}
\text { Rate of mass } \\
\text { accumulation }
\end{array}\right\}=\left\{\begin{array}{c}
\text { Rate of } \\
\text { mass in }
\end{array}\right\}-\left\{\begin{array}{l}
\text { Rate of } \\
\text { mass out }
\end{array}\right\} .
$$

For the faces of the volume element indicated by the numbers " 1 " and " 2 " we may write that the rate of mass in at $r$ is

$$
\left.\mathrm{r} \Delta \theta \Delta \mathrm{z \rho u}\right|_{\mathbf{r}}
$$

and the rate of mass out at $r+\Delta r$ is

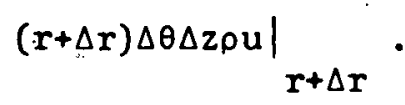

For the faces indicated by the numbers " 3 ". and " 4 ", we may write that the rate of mass in at $\theta$ is

$$
\left.\Delta \mathrm{r} \Delta \mathrm{z \rho v}\right|_{\theta}
$$

and the rate of mass out at $\theta+\Delta \theta$ is

$$
\left.\Delta r \Delta z \rho v\right|_{\theta+\Delta \theta}
$$

Finally, for the faces indicated by the numbers "5" and "6", we write that the rate of mass in at $z$ is 


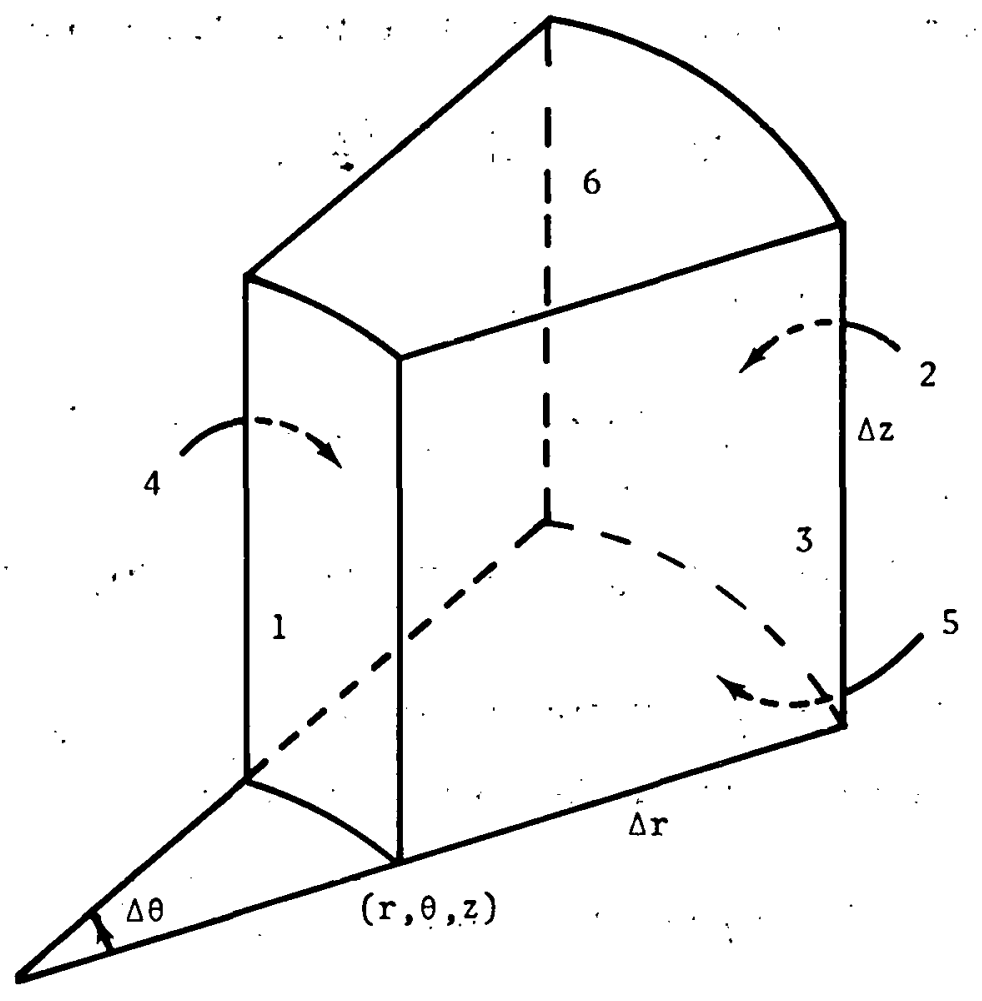

Figure 2. Volume Element in Cylindrical Coordinates. 


$$
\left.\left(\mathrm{r}+\frac{1}{2} \Delta \mathrm{r}\right) \Delta \mathrm{r} \Delta \theta \rho \mathrm{w}\right|_{\mathrm{z}},
$$

and the rate of mass out at $z+\Delta z$ is

$$
\left.\left(\mathbf{r}+\frac{1}{2} \Delta r\right) \Delta r \Delta \theta \rho w\right|_{z+\Delta z}
$$

The rate of mass accumulation within the volume element is

$$
\left(\mathbf{r}+\frac{1}{2} \Delta \mathbf{r}\right) \Delta r \Delta \theta \Delta z \frac{\partial \rho}{\partial t}
$$

and Equation (2.4) may be written as

$$
\begin{aligned}
& \left(\mathbf{r}+\frac{1}{2} \Delta r\right) \Delta r \Delta \theta \Delta z \frac{\partial \rho}{\partial t}=\Delta \theta \Delta z\left\{\left.r \rho u\right|_{r}-\left.(r+\Delta r) \rho u\right|_{r+\Delta r}\right\} \\
& +\Delta r \Delta z\left\{\left.\rho v\right|_{\theta}-\left.\rho v\right|_{\theta+\Delta \theta}\right\}+\left(r+\frac{1}{2} \Delta r\right) \Delta r \Delta \theta\left\{\left.\rho w\right|_{z}-\left.\rho w\right|_{z+\Delta z}\right\} \text {. }
\end{aligned}
$$

If we divide both sides of Equation (2.5) by $\Delta \mathrm{r} \Delta \theta \Delta \mathrm{z}$ and take the limit as $\Delta r, \Delta \theta$ and $\Delta z$ approach zero simultaneously, we obtain

$$
\mathbf{r} \frac{\partial \rho}{\partial t}=-\frac{\partial}{\partial \mathbf{r}}(\rho \mathrm{ru})-\frac{\partial}{\partial \theta}(\rho \mathbf{v})-\mathbf{r} \frac{\partial}{\partial z}(\rho w),
$$

which may be written as

$$
\frac{\partial \rho}{\partial t}+\frac{1}{r} \frac{\partial}{\partial r}(\rho r u)+\frac{1}{r} \frac{\partial}{\partial \theta}(\rho v)+\frac{\partial}{\partial z}(\rho w)=0
$$

This is the "equation of continuity" and describes the rate of change of density at a fixed point resulting from changes in the mass velocity vector $\rho \vec{v}$. 
For the same volume element conservation of momentum may be written as the momentum balance

$$
\left\{\begin{array}{c}
\text { Rate of } \\
\text { momentum } \\
\text { accumulation }
\end{array}\right\}=\left\{\begin{array}{c}
\text { Rate of } \\
\text { momentum } \\
\text { in }
\end{array}\right\}-\left\{\begin{array}{c}
\text { Rate of } \\
\text { momentum } \\
\text { out }
\end{array}\right\}+\left\{\begin{array}{c}
\text { Sum of } \\
\text { forces acting } \\
\text { on system }
\end{array}\right\}
$$

Since we allow the fluid to move through all six faces of the volume element in any direction as in Figure 3, we note that Equation (2.7) is a vector equation with components in each of the coordinate directions $r$, $\theta$ and $z$. Let us consider the r-component of each term in Equation (2.7). Momentum flows into and out of the volume element by convection (i.e., bulk fluid flow) and by molecular transfer (i.e., velocity gradients). The rate at which the $r$-component of momentum enters the face at $r$ by convection is

$$
\left.\mathrm{r} \Delta \theta \Delta \mathrm{z}(\rho \mathrm{u}) \mathrm{u}\right|_{\mathbf{r}}
$$

and the rate at which it leaves at $r+\Delta r$ is

$$
\left.(\mathrm{r}+\Delta \mathrm{r}) \Delta \theta \Delta \mathbf{z}(\rho \mathrm{u}) \mathrm{u}\right|_{\mathbf{r}+\Delta \mathrm{r}}
$$

There are analagous expressions for the $r$-component of convective momentum across the other four faces. In addition, fluid motion in the $\theta-$ direction results in an effective force in the r-direction, the "centrifugal force," given by 


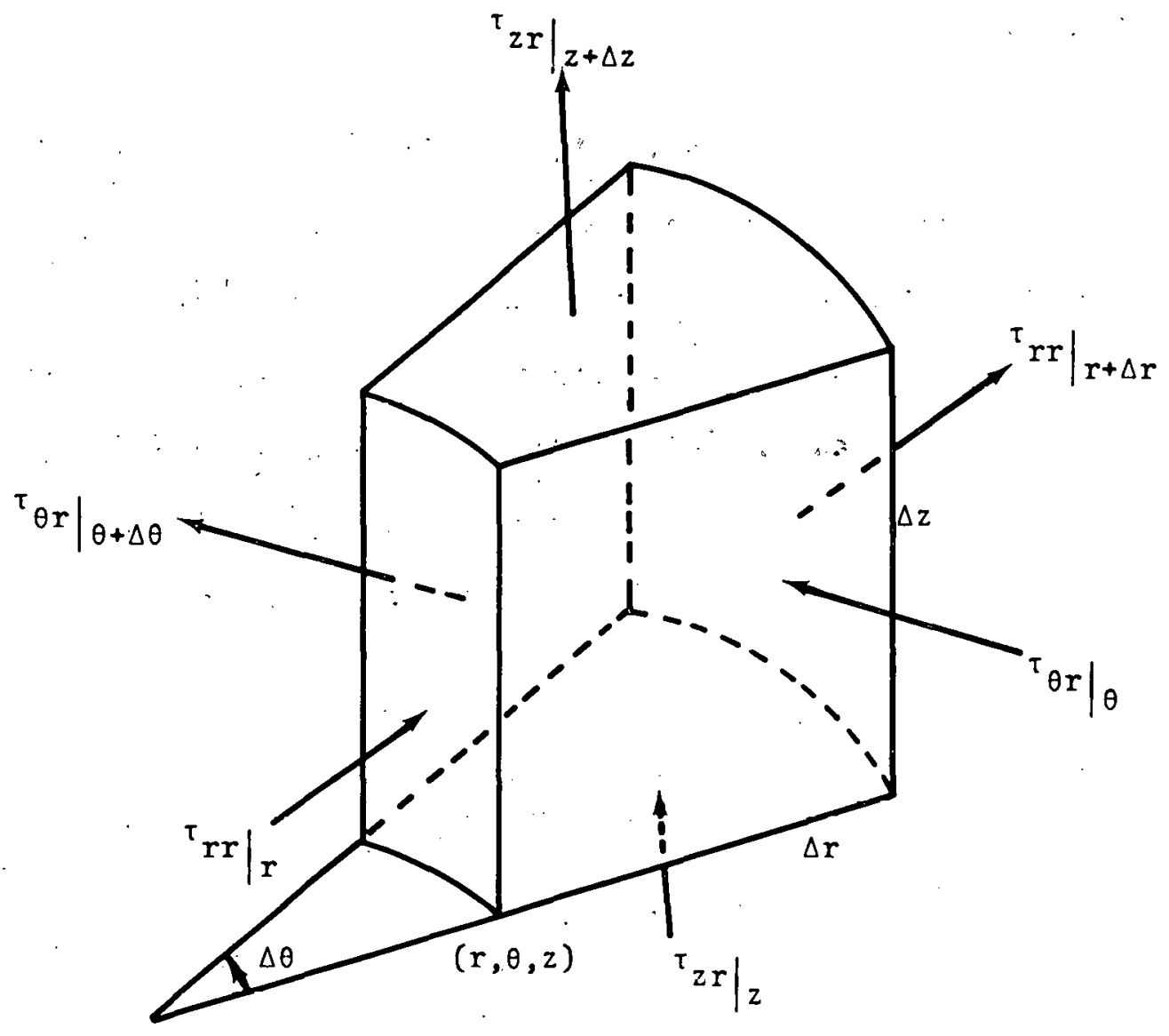

Figure 3. Directions in which the R-Component of Momentum is Transported Through a Volume Element. 
We find that the net convective r-momentum flowing into the volume element is

$$
\begin{gathered}
\left.\Delta \theta \Delta z \text { foruu }_{\mathbf{r}}-\left.\rho(\mathrm{r}+\Delta \mathrm{r}) \mathrm{uu}\right|_{\mathrm{r}+\Delta \mathrm{r}}\right\}+\Delta \mathrm{r} \Delta \mathrm{z}\left\{\left.\rho \mathrm{vu}\right|_{\theta}-\left.\rho v u\right|_{\theta+\Delta \theta}\right\} \\
+\left(\mathrm{r}+\frac{1}{2} \Delta \mathrm{r}\right) \Delta \mathrm{r} \Delta \theta\left\{\left.\rho w u\right|_{z}-\left.\rho w u\right|_{z+\Delta z}\right\}+\Delta r \Delta \theta \Delta z \rho v v
\end{gathered}
$$

Similarly, the rate at which the $r$-component of momentum enters the face at $\mathbf{r}$ by molecular transport is

$$
\left.\mathbf{r} \Delta \theta \Delta z \tau_{\mathbf{r r}}\right|_{\mathbf{r}}
$$

and the rate at which it leaves at $r+\Delta r$ is

$$
\left.(r+\Delta r) \Delta \theta \Delta z \tau_{r r}\right|_{r+\Delta r}
$$

Analagous to the centrifugal force discussed above, molecular transport in the $\theta$-direction results in an effective force in the $r$-direction which is given by

$$
\Delta \mathbf{r} \Delta \theta \Delta \mathbf{z} \tau_{\theta \theta}
$$

When the $r$-components of molecular transport momentum are computed for the other four faces of the volume element, we find that the net molecular transport $r$-momentum flowing into the element is

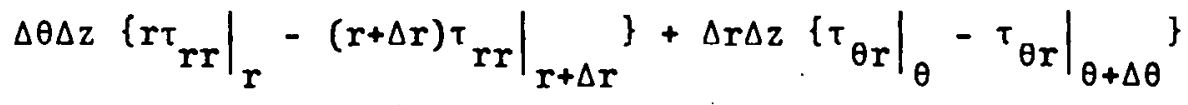

$$
\begin{aligned}
& +\left(\mathbf{r}+\frac{1}{2} \Delta \mathrm{r}\right) \Delta \mathrm{r} \Delta \theta\left\{\left.\tau_{z \mathrm{r}}\right|_{z}-\left.\tau_{z r}\right|_{z+\Delta z}\right\}+\Delta \mathrm{r} \Delta \theta \Delta z \tau_{\theta \theta} .
\end{aligned}
$$


In many cases the only important external forces acting on the system are those arising from the fluid pressure $P$ defined by the equation of state,

$$
P .=P(\rho, T),
$$

where $T$ is the temperature and

$$
\rho=\rho(r, \theta, z, t),
$$

and the gravitational force per unit mass

$$
\vec{g}=\vec{g}(r, \theta, z)
$$

Since we will later assume that the gravitational force has only a negligible effect on the physicai system of interest, we will omit it from the remainder of the discussion. The change in the force due to the pressures at $\mathbf{r}$ and at $\mathbf{r}+\Delta \mathbf{r}$ is

$$
\Delta \theta \Delta z\left\{\left.\mathbf{r P}\right|_{\mathbf{r}}-\left.(\mathbf{r}+\Delta \mathbf{r}) \mathbf{P}\right|_{\mathbf{r}+\Delta \mathbf{r}}\right\}
$$

and the pressures normal to the surfaces at $\theta$ and at $\theta+\Delta \theta$ result in a pressure force in the r-direction given by

$$
2 \cdot \Delta r \Delta z \cdot P \sin \left(\frac{1}{2} \Delta \theta\right)
$$

Now we may write the r-component of the external forces acting on the system as

$$
\Delta \theta \Delta z\left\{\left.r P\right|_{r}-\left.(r+\Delta r) P\right|_{r+\Delta r}\right\}+2 \Delta r \Delta z P \sin \left(\frac{1}{2} \Delta \theta\right)
$$


Finally, the rate of accumulation of $r$-momentum is

$$
\left(\mathbf{r}+\frac{1}{2} \Delta r\right) \Delta r \Delta \theta \Delta z \frac{\partial}{\partial t}(\rho u),
$$

and we may write the r-component of Equation (2.7) as

$$
\begin{aligned}
& \left(r+\frac{1}{2} \Delta r\right) \Delta r \Delta \theta \Delta z \frac{\partial}{\partial t}(\rho u)=\Delta \theta \Delta z\left\{\left.\rho r u u\right|_{r}-\left.\rho(r+\Delta r) u u\right|_{r+\Delta r}\right\} \\
& +\Delta r \Delta z\left\{\left.\rho v u\right|_{\theta}-\left.\rho v u\right|_{\theta+\Delta \theta}\right\}+\left(r+\frac{1}{2} \Delta r\right) \Delta r \Delta \theta\left\{\left.\rho w u\right|_{z}-\left.\rho w u\right|_{z+\Delta z}\right\} \\
& +\Delta r \Delta \theta \Delta z \rho v v+\Delta \theta \Delta z\left\{\left.r P\right|_{r}-\left.(r+\Delta r) P\right|_{r+\Delta r}\right\}+2 \Delta r \Delta z P \sin \left(\frac{1}{2} \Delta \theta\right) \\
& +\Delta \theta \Delta z\left\{\left.r \tau \operatorname{rr}\right|_{r}-\left.(r+\Delta r) \tau \operatorname{rr}\right|_{r+\Delta r}\right\}+\Delta r \Delta z\left\{\left.\tau_{\theta r}\right|_{\theta}-\left.\tau_{\theta r}\right|_{\theta+\Delta \theta}\right\} \\
& +\left(\mathrm{r}+\frac{1}{2} \Delta \mathrm{r}\right) \Delta \mathrm{r} \Delta \theta\left\{\left.\tau_{\mathrm{zr}}\right|_{z}-\left.\tau_{z \mathrm{r}}\right|_{\mathrm{z}+\Delta z}\right\}+\Delta \mathrm{r} \Delta \theta \Delta z \tau_{\theta \theta} .
\end{aligned}
$$

If we divide Equation (2.8) by $\Delta r \Delta \theta \Delta z$ and take the limit as these quantities approach zero simultaneously, we obtain

$$
\begin{aligned}
& r \frac{\partial}{\partial t}(\rho u)=-\frac{\partial}{\partial r}(\rho r u u)-\frac{\partial}{\partial \theta}(\rho v u)-r \frac{\partial}{\partial z}(\rho w u)+\rho v v-\frac{\partial}{\partial r}(r P) \\
&+P-\frac{\partial}{\partial r}\left(r \tau_{r r}\right)-\frac{\partial}{\partial \theta}\left(\tau_{\theta r}\right)-r_{\frac{\partial}{\partial z}}\left(\tau_{z r}\right)+\tau_{\theta \theta} .
\end{aligned}
$$

We now divide Equation (2.9) by $r$, perform portions of the indicated differentiation, and collect terms to obtain 


$$
\begin{aligned}
& u\left\{\frac{\partial \rho}{\partial t}+\frac{1}{r} \frac{\partial}{\partial r}(\rho r u)+\frac{1}{r} \frac{\partial}{\partial \theta}(\rho v)+\frac{\partial}{\partial z}(\rho w)\right\} \\
& \quad+\rho\left\{\frac{\partial u}{\partial t}+u \frac{\partial u}{\partial r}+\frac{v}{r} \frac{\partial u}{\partial \theta}+w \frac{\partial u}{\partial z}-\frac{v^{2}}{r}\right\} \\
& \quad=-\frac{\partial P}{\partial r}-\left\{\frac{1}{r} \frac{\partial}{\partial r}\left(r \tau_{r}\right)+\frac{1}{r} \frac{\partial}{\partial \theta}\left(\tau_{\theta r}\right)-\frac{\tau_{\theta \theta}}{r}+\frac{\partial}{\partial z}\left(\tau_{z r}\right)\right\} .
\end{aligned}
$$

We recognize the first term of Equation (2.10) as the left member of equation of continuity, Equation (2.6), hence the $r$-component is

$$
\begin{aligned}
& \rho\left\{\frac{\partial u}{\partial t}+u \frac{\partial u}{\partial r}+\frac{v}{r} \frac{\partial u}{\partial \theta}+w \frac{\partial u}{\partial z}-\frac{v^{2}}{r}\right\} \\
& =-\frac{\partial P}{\partial r}-\left\{\frac{1}{r} \frac{\partial}{\partial r}\left(r_{r r}\right)+\frac{1}{r} \frac{\partial}{\partial \theta}\left(\tau_{\theta r}\right)-\frac{\tau_{\theta \theta}}{r}+\frac{\partial}{\partial z}\left(\tau_{z r}\right)\right\}
\end{aligned}
$$

Through similar analyses we obtain the following expressions for the $\theta$ - and $z$-components of Equation (2.7)

$$
\begin{aligned}
\rho & \left\{\frac{\partial v}{\partial t}+u \frac{\partial v}{\partial r}+\frac{v}{r} \frac{\partial v}{\partial \theta}+\frac{u v}{r}+w \frac{\partial v}{\partial z}\right\}=-\frac{1}{r} \frac{\partial P}{\partial \theta} \\
- & \left\{\frac{1}{r^{2}} \frac{\partial}{\partial r}\left(r^{2} \tau_{r \theta}\right)+\frac{1}{r} \frac{\partial}{\partial \theta}\left(\tau_{\theta \theta}\right)+\frac{\partial}{\partial z}\left(\tau_{z \theta}\right)\right\},
\end{aligned}
$$

and

$$
\begin{aligned}
& \rho\left\{\frac{\partial w}{\partial t}+u \frac{\partial w}{\partial r}+\frac{v}{r} \frac{\partial w}{\partial \theta}+w \frac{\partial w}{\partial z}\right\}=-\frac{\partial p}{\partial z} \\
& -\left\{\frac{1}{r} \frac{\partial}{\partial r}\left(r \tau_{r z}\right)+\frac{1}{r} \frac{\partial}{\partial \theta}\left(\tau_{\theta z}\right)+\frac{\partial}{\partial z}\left(\tau_{z z}\right)\right\} .
\end{aligned}
$$


For the physical problem in which we are presently interested (and indeed, for a large number of physical problems) the density $\rho$ and the viscosity $\mu$ can be considered constant." Under this assumption the equation of continuity, Equation (2.6), may be written as

$$
\frac{1}{r} \frac{\partial}{\partial r}(r u)+\frac{1}{r} \frac{\partial v}{\partial \theta}+\frac{\partial w}{\partial z}=0
$$

the continuity equation for incompressible fluids. Since it is our purpose to extract information regarding the velocity distributions from Equations (2.11) - (2.13), we must replace the various stresses in these equations with expressions in terms of velocity gradients and the viscosity of the fluid. For incompressible Newtonian fluids with constant viscosity these expressions (from Bird, Stewart and Lightfoot [1]) are given in Table I. Upon the substitution of these expressions into. Equations (2.11) - (2.13), we obtain the $r-, \theta$ - and $z$-components of the Navier-Stokes equation, given by

$$
\begin{aligned}
& \frac{\partial u}{\partial t}+u \frac{\partial u}{\partial r}+\frac{v}{r} \frac{\partial u}{\partial \theta}-\frac{v^{2}}{r}+w \frac{\partial u}{\partial z}=-\frac{1}{\rho} \frac{\partial p}{\partial r} \\
& +\frac{\mu}{\rho}\left\{\frac{\partial}{\partial r}\left(\frac{1}{r} \frac{\partial}{\partial r}(r u)\right)+\frac{1}{r^{2}} \frac{\partial^{2} u}{\partial \theta^{2}}-\frac{2}{r^{2}} \frac{\partial v}{\partial \theta}+\frac{\partial^{2} u}{\partial z^{2}}\right\}, \\
& \frac{\partial v}{\partial t}+u \frac{\partial v}{\partial r}+\frac{v}{r} \frac{\partial v}{\partial \theta}+\frac{u v}{r}+w \frac{\partial v}{\partial z}=-\frac{1}{\rho r} \frac{\partial p}{\partial \theta} \\
& +\frac{\mu}{\rho}\left[\frac{\partial}{\partial r}\left(\frac{1}{r} \frac{\partial}{\partial r}(r v)\right)+\frac{1}{r^{2}} \frac{\partial^{2} v}{\partial \theta^{2}}+\frac{2}{r^{2}} \frac{\partial u}{\partial \theta}+\frac{\partial^{2} v}{\partial z^{2}}\right\},
\end{aligned}
$$

and 
TABLE I

STRESSES IN TERMS OF VELOCITY GRADIENTS AND

VISCOSITY FOR. INCOMPRESSIBLE

NEWTONIAN FLUIDS

$$
\begin{aligned}
& \tau_{r r}=-2 \mu \frac{\partial u}{\partial r} \\
& \tau_{\theta \theta}=-2 \mu\left(\frac{1}{r} \frac{\partial v}{\partial \theta}+\frac{u}{r}\right) \\
& \tau_{z z}=-2 \mu \frac{\partial w}{\partial z} \\
& \tau_{r \theta}=\tau_{\theta r}=-\mu\left(r \frac{\partial}{\partial r}\left(\frac{v}{r}\right)+\frac{1}{r} \frac{\partial u}{\partial \theta}\right) \\
& \tau_{\theta z}=\tau_{z \theta}=-\mu\left(\frac{\partial v}{\partial z}+\frac{1}{r} \frac{\partial w}{\partial \theta}\right) \\
& \tau_{z r}=\tau_{r z}=-\mu\left(\frac{\partial w}{\partial r}+\frac{\partial u}{\partial z}\right)
\end{aligned}
$$




$$
\begin{aligned}
\frac{\partial w}{\partial t} & +u \frac{\partial w}{\partial r}+\frac{v}{r} \frac{\partial w}{\partial \theta}+w \frac{\partial w}{\partial z}=-\frac{1}{\rho} \frac{\partial p}{\partial z} \\
& +\frac{\mu}{\rho}\left\{\frac{1}{r} \frac{\partial}{\partial r}\left(r \frac{\partial w}{\partial r}\right)+\frac{1}{r^{2}} \frac{\partial^{2} w}{\partial \theta^{2}}+\frac{\partial^{2} w}{\partial z}\right\}
\end{aligned}
$$

It should be noted that Equations (2.14) - (2.17) are four partial differential equations in the four dependent variables $u, v, w$ and $P$ which are functions of the four independent variables $r, \theta, z$ and $t$. Equation (2.14) is a linear first order partial differential equation, and Equations $(2.15)-(2.17)$ are second order semi-linear elliptic partial differential equations.

Until now the equations that have been developed describe incompressible flow situations in which the effects due to the gravitational force are negligible. For reasons which will become apparent later, we now limit the discussion to problems which possess axial symmetry, i.e., no $\theta$-dependence. We also seek to rephrase Equations $(2.14)-(2.17)$ in terms of the classical stream function $\psi$ and the vorticity $\zeta$. First, define the function $\Gamma$ by

$$
r(r, z, t) \equiv r v(r, z, t)
$$

and recall the definition of the kinematic viscosity given by Equation (2.3). Then Equations (2.14) - (2.17) can be written as

$$
\frac{1}{r} \frac{\partial}{\partial r}(r u)+\frac{\partial w}{\partial z}=0
$$




$$
\begin{aligned}
& \frac{\partial u}{\partial t}+u \frac{\partial u}{\partial r}-\frac{\Gamma^{2}}{r^{3}}+w \frac{\partial u}{\partial z}=-\frac{1}{\rho} \frac{\partial p}{\partial r}+v\left\{\frac{\partial}{\partial r}\left(\frac{1}{r} \frac{\partial}{\partial r}(r u)\right)+\frac{\partial^{2} u}{\partial z^{2}}\right\}, \\
& \frac{\partial \Gamma}{\partial t}+u \frac{\partial \Gamma}{\partial r}+w \frac{\partial \Gamma}{\partial z}=v\left\{r \frac{\partial}{\partial r}\left(\frac{1}{r} \frac{\partial \Gamma}{\partial r}\right)+\frac{\partial^{2} \Gamma}{\partial z^{2}}\right\} \\
& \frac{\partial w}{\partial t}+u \frac{\partial w}{\partial r}+w \frac{\partial w}{\partial z}=-\frac{1}{\rho} \frac{\partial p}{\partial z}+v\left\{\frac{1}{r} \frac{\partial}{\partial r}\left(r \frac{\partial w}{\partial r}\right)+\frac{\partial^{2} w}{\partial z^{2}}\right\} .
\end{aligned}
$$

We can eliminate the pressure $P$ from these equations by differentiating Equation (2.20) with respect to $z$ and Equation (2.22) with respect to $r$ and by subtracting the results to obtain

$$
\begin{aligned}
\frac{\partial}{\partial t}\left[\frac{\partial u}{\partial z}-\frac{\partial w}{\partial r}\right]+\frac{\partial u}{\partial r}\left[\frac{\partial u}{\partial z}-\frac{\partial w}{\partial r}\right]+u \frac{\partial}{\partial r}\left[\frac{\partial u}{\partial z}-\frac{\partial w}{\partial r}\right]+\frac{\partial w}{\partial z}\left[\frac{\partial u}{\partial z}-\frac{\partial w}{\partial r}\right]+w \frac{\partial}{\partial z}\left[\frac{\partial u}{\partial z}-\frac{\partial w}{\partial r}\right] \\
-\frac{2}{r} \Gamma \frac{\partial \Gamma}{\partial z}=v\left[\frac{\partial^{2}}{\partial r}\left[\frac{\partial u}{\partial z}-\frac{\partial w}{\partial r}\right]+\frac{1}{r} \frac{\partial}{\partial r}\left[\frac{\partial u}{\partial z}-\frac{\partial w}{\partial r}\right]-\frac{1}{r^{2}}\left[\frac{\partial u}{\partial z}-\frac{\partial w}{\partial r}\right]\right. \\
\left.+\frac{\partial^{2}}{\partial z^{2}}\left[\frac{\partial u}{\partial z}-\frac{\partial w}{\partial r}\right]\right\}
\end{aligned}
$$

We define the vorticity $\zeta$ by

$$
\zeta(r, z, t) \equiv \frac{\partial u}{\partial z}-\frac{\partial w}{\partial r},
$$

and note that, for any constant $C$,

$$
C \frac{\partial u}{\partial r}+C \frac{\partial w}{\partial z}=-C \frac{u}{r} \text {. }
$$

from Equation (2.19). Now Equation (2.23) may be written as 
$\frac{\partial \zeta}{\partial t}+u \frac{\partial \zeta}{\partial r}+w \frac{\partial \zeta}{\partial z}-\frac{u \zeta}{r}-2 \frac{\Gamma}{r^{3}} \frac{\partial \Gamma}{\partial z}=v\left\{\frac{\partial^{2} \zeta}{\partial r^{2}}+\frac{1}{r} \frac{\partial \zeta}{\partial r}-\frac{\zeta}{r^{2}}+\frac{\partial^{2} \zeta}{\partial z^{2}}\right\}$

To express Equations (2.19), (2.21) and (2.26) in terms of nondimensional quantities we define the variables.

$$
\begin{aligned}
& r^{\prime}=r / L, \\
& z^{\prime}=z / L, \\
& u^{\prime}=u / L \Omega, \\
& v^{\prime}=v / L \Omega, \\
& w^{\prime}=w / L \Omega,
\end{aligned}
$$

and

$$
t^{\prime}=\Omega t,
$$

where $L$ is some (problem dependent) characteristic length and $\Omega$ is some characteristic angular velocity. We note that the equation of continuity, Equation (2.19), in terms of these variables is identically satisfied, for

$$
\frac{\partial u^{\prime}}{\partial r^{\prime}}+\frac{u^{\prime}}{r^{\prime}}+\frac{\partial w^{\prime}}{\partial z^{\prime}} \equiv \Omega^{-1}\left(\frac{\partial u}{\partial r}+\frac{u}{r}+\frac{\partial w}{\partial z}\right) \equiv 0
$$

In terms of the nondimensional variables Equation (2.21) is

$$
\frac{\partial \Gamma^{\prime}}{\partial t^{\prime}}+u^{\prime} \frac{\partial \Gamma^{\prime}}{\partial r^{\prime}}+w^{\prime} \frac{\partial \Gamma^{\prime}}{\partial z^{\prime}}=\frac{\nu}{L^{2} \Omega}\left\{r^{\prime} \frac{\partial}{\partial r^{\prime}}\left(\frac{1}{r^{\prime}} \frac{\partial \Gamma^{\prime}}{\partial r^{\prime}}\right)+\frac{\partial^{2} \Gamma^{\prime}}{\partial z^{\prime 2}}\right\},
$$


and Equation (2.26) may be written as

$$
\begin{gathered}
\frac{\partial \zeta^{\prime}}{\partial t^{\prime}}+u^{\prime} \frac{\partial \zeta^{\prime}}{\partial r^{\prime}}+w^{\prime} \frac{\partial \zeta^{\prime}}{\partial z^{\prime}}-\frac{u^{\prime} \zeta^{\prime}}{r^{\prime}}-2 \frac{\Gamma^{\prime}}{r^{\prime 3}} \frac{\partial \Gamma^{\prime}}{\partial z^{\prime}} \\
=\frac{v}{L^{2} \Omega}\left\{\frac{\partial^{2} \zeta^{\prime}}{\partial r^{\prime 2}}+\frac{1}{r^{\prime}} \frac{\partial \zeta^{\prime}}{\partial r^{\prime}}-\frac{\zeta^{\prime}}{r^{\prime 2}}+\frac{\partial^{2} \zeta^{\prime}}{\partial z^{\prime 2}}\right\} .
\end{gathered}
$$

We now introduce a dimensionless stream function $\psi^{\prime}=\psi^{\prime}\left(r^{\prime}, z^{\prime}, t^{\prime}\right)$

defined by

$$
u^{\prime}=\frac{1}{r^{\prime}} \frac{\partial \psi^{\prime}}{\partial z^{\prime}}, \quad w^{\prime}=-\frac{1}{r^{\prime}} \frac{\partial \psi^{\prime}}{\partial r^{\prime}}
$$

Note that the equation of continuity, Equation (2.28), is identically satisfied, for

$\frac{\partial u^{\prime}}{\partial r^{\prime}}+\frac{u^{\prime}}{r^{\prime}}+\frac{\partial w^{\prime}}{\partial z^{\prime}}=\frac{1}{r^{\prime}} \frac{\partial^{2} \psi^{\prime}}{\partial r^{\prime} \partial z^{\prime}}-\frac{1}{r^{\prime}} \frac{\partial \psi^{\prime}}{\partial z^{\prime}}+\frac{1}{r^{\prime 2}} \frac{\partial \psi^{\prime}}{\partial z^{\prime}}-\frac{1}{r^{\prime}} \frac{\partial^{2} \psi^{\prime}}{\partial r^{\prime} \partial z^{\prime}} \equiv 0$

We define the dimensionless tangential Reynolds number $\operatorname{Re}$ by

$$
\operatorname{Re}=\frac{L_{\Omega}^{2}}{v}
$$

Now Equation $(2.28)$ is

$$
\frac{\partial \Gamma^{\prime}}{\partial t^{\prime}}+\frac{1}{r^{\prime}} \frac{\partial \psi^{\prime}}{\partial z^{\prime}} \frac{\partial \Gamma^{\prime}}{\partial r^{\prime}}-\frac{1}{r^{\prime}} \frac{\partial \psi^{\prime}}{\partial r^{\prime}} \frac{\partial \Gamma^{\prime}}{\partial z^{\prime}}=\frac{1}{R e}\left\{\Delta \Gamma^{\prime}=-\frac{1}{r^{\prime}} \frac{\partial \Gamma^{\prime}}{\partial r^{\prime}}\right\}
$$


Equation (2.29) is

$$
\begin{gathered}
\frac{\partial \zeta^{\prime}}{\partial t^{\prime}}+\frac{1}{r^{\prime}} \frac{\partial \psi^{\prime}}{\partial z^{\prime}} \frac{\partial \zeta^{\prime}}{\partial r^{\prime}}-\frac{1}{r^{\prime}} \frac{\partial \psi^{\prime}}{\partial r^{\prime}} \frac{\partial \zeta^{\prime}}{\partial z^{\prime}}-\frac{1}{r^{\prime 2}} \zeta^{\prime} \frac{\partial \psi^{\prime}}{\partial z^{\prime}}-\frac{2}{r^{\prime 3}} \Gamma^{\prime} \frac{\partial \Gamma^{\prime}}{\partial z^{\prime}} \\
=\frac{1}{\operatorname{Re}}\left\{\Delta \zeta^{\prime}+\frac{1}{r^{\prime}} \frac{\partial \zeta^{\prime}}{\partial r^{\prime}}-\frac{1}{r^{\prime 2}} \zeta^{\prime}\right\},
\end{gathered}
$$

and Equation $(2.24)$ is

$$
\Delta \psi^{\prime}-\frac{1}{r^{\prime}} \frac{\partial \psi^{\prime}}{\partial r^{\prime}}=r^{\prime} \zeta^{\prime}
$$

where

$$
\Delta \equiv \frac{\partial^{2}}{\partial \mathrm{r}^{\prime 2}}+\frac{\partial^{2}}{\partial z^{\prime 2}} .
$$

We will drop the primes in Equations (2.34) - (2.36) and will remember that any boundary and initial conditions must be expressed in terms of the dimensionless quantities. We have the three second order semi-linear partial differential equations

$$
\begin{gathered}
\frac{\partial \Gamma}{\partial t}+\frac{1}{r} \frac{\partial \psi}{\partial z} \frac{\partial \Gamma}{\partial r}-\frac{1}{r} \frac{\partial \psi}{\partial r} \frac{\partial \Gamma}{\partial z}=\frac{1}{\operatorname{Re}}\left\{\Delta \Gamma-\frac{1}{r} \frac{\partial \Gamma}{\partial r}\right\}, \\
\frac{\partial \zeta}{\partial t}+\frac{1}{r} \frac{\partial \psi}{\partial z} \frac{\partial \zeta}{\partial r}-\frac{1}{r} \frac{\partial \psi}{\partial r} \frac{\partial \zeta}{\partial z}-\frac{1}{r^{2}} \zeta \frac{\partial \psi}{\partial z}-\frac{2}{r} \Gamma \frac{\partial \Gamma}{\partial z} \\
=\frac{1}{\operatorname{Re}}\left\{\Delta \zeta+\frac{1}{r} \frac{\partial \zeta}{\partial r}-\frac{1}{r^{2}} \zeta\right\},
\end{gathered}
$$

and 


$$
\Delta \psi-\frac{1}{r} \frac{\partial \psi}{\partial r}=r \zeta
$$

where

$$
\Delta \equiv \frac{\partial^{2}}{\partial r^{2}}+\frac{\partial^{2}}{\partial z^{2}}
$$

We have developed two systems of partial differential equations (one given by Equations (2.19) - (2.22) and the other by Equations (2.37) (2.39)) which describe axisymmetric, incompressible flow problems in which the effects due to the gravitational force are negligible. 
A reactor concept involving an annular cloud of fissionable material suspended by a vortex flow in a cylindrical container has been of interest to the Oak Ridge National Laboratory because of its propulsion and power generation applications. This concept has been studied by Kerrebrock and Keyes [2], Kerrebrock and Meghreblian [3] and Keyes [4]. As a result of the experimental observations of Kenda11 [5], and Rosenzweig, Lewellen and Ross [6], it was decided that it was necessary to examine in detail the interaction of this axisymmetric vortex flow with the end walls of the containing cylinder. As described in his dissertation, Kidd [7] simulated such a reactor with a cylinder (containing water) the walls of which were in rotation with a constant angular velocity while one of the cylinder end walls remained stationary. Fluid was passed into the cylinder in the radial direction along the outside cylinder wall and was allowed to pass out of the cylinder at a porous center tube along the axis of the cylinder. Experimental measurements of the velocity distributions near the stationary end wall were obtained.

After an attempt was made to determine these velocity distributions from the equations of motion by means of a similarity transformation (to be discussed in Chapter $V$ ), it was decided that an effort should be made to solve the equations of motion numerically. Such an approach seemed plausible for, since the advent of large-memory, high-speed digital computers, a number of flow problems have been successfully 
investigated by numerical methods. In 1963, Fromm [8] proposed a numerical method for treating transient flow problems and, in particular, examined the development of the Karman vortex street. In an expository paper, Pearson [9] discussed the stability of a numerical technique for solving a fourth-order partial differential equation involving the classical stream function. Then, Pearson $[10,11]$ used this technique to solve problems which included vortex formation and flow between rotating, coaxial disks. In a dissertation, Tejeira [12] discussed the use of a numerical technique to solve equations analogous to Equations $(2.36)-(2.38)$. It should be noted that the papers mentioned above are all concerned with two-dimensional, incompressible, transient flow problems.

As an initial step toward finding a numerical solution we examine a problem.similar to that of Kidd except that there is no flow into or . out of the cylinder. For the present work we seek solutions of this problem at steady-state.

In terms of the velocity components and the pressure, the partial differential equations (from Equations (2.19) - (2.22)) are

$$
\begin{gathered}
\frac{1}{r} \frac{\partial}{\partial r}(r u)+\frac{\partial w}{\partial z}=0, \\
u \frac{\partial u}{\partial r}-\frac{v^{2}}{r}+w \frac{\partial u}{\partial z}=-\frac{1}{\rho} \frac{\partial p}{\partial r}+v\left\{\frac{\partial}{\partial r}\left(\frac{1}{r} \frac{\partial}{\partial r}(r u)\right)+\frac{\partial^{2} u}{\partial z^{2}}\right\}, \\
u \frac{\partial v}{\partial r}+\frac{u v}{r}+w \frac{\partial v}{\partial z}=v\left\{\frac{\partial}{\partial r}\left(\frac{1}{r} \frac{\partial}{\partial r}(r v)\right)+\frac{\partial^{2} v}{\partial z^{2}}\right\},
\end{gathered}
$$

and 


$$
u \frac{\partial w}{\partial r}+w \frac{\partial w}{\partial z}=-\frac{1}{\rho} \frac{\partial P}{\partial z}+v\left\{\frac{1}{r} \frac{\partial}{\partial r}\left(r \frac{\partial w}{\partial r}\right)+\frac{\partial^{2} w}{\partial z^{2}}\right\}
$$

with boundary conditions for the velocity components given by

$$
\begin{aligned}
& u\left(R_{0}, z\right)=w\left(R_{0}, z\right)=0, v\left(R_{0}, z\right)=R_{0} \Omega, \text { for } 0 \leq z \leq L, \\
& u\left(R_{I}, z\right)=w\left(R_{I}, z\right)=0, v\left(R_{I}, z\right)=R_{I} \Omega, \text { for } 0 \leq z \leq L, \\
& u(r, 0)=w(r, 0)=0, v(r, 0)=r \Omega, \text { for } R_{I} \leq r \leq R_{0},
\end{aligned}
$$

and

$$
u(r, L)=v(r, L)=w(r, L)=0 \text {, for } R_{I} \leq r \leq R_{0} \text {, }
$$

where $R_{0}$ is the radius of the containing cylinder, $\Omega$ is the constant angular velocity of the containing cylinder, $L$ is the height of the cylinder, and $R_{I}$ is the radius of the center tube.

In terms of the dimensionless stream function and vorticity, the partial differential equations (from Equations (2.37) - (2.39)) are

$$
\begin{gathered}
\frac{1}{r} \frac{\partial \psi}{\partial z} \frac{\partial \Gamma}{\partial r}-\frac{1}{r} \frac{\partial \psi}{\partial r} \frac{\partial \Gamma}{\partial z}=\frac{1}{R e}\left\{\Delta \Gamma-\frac{1}{r} \frac{\partial \Gamma}{\partial r}\right\}, \\
\frac{1}{r} \frac{\partial \psi}{\partial z} \frac{\partial \zeta}{\partial r}-\frac{1}{r} \frac{\partial \psi}{\partial r} \frac{\partial \zeta}{\partial z}-\frac{1}{r^{2}} \zeta \frac{\partial \psi}{\partial z}-\frac{2}{r} \Gamma \frac{\partial \Gamma}{\partial z} \\
=\frac{1}{\operatorname{Re}}\left\{\Delta \zeta+\frac{1}{r} \frac{\partial \zeta}{\partial r}-\frac{1}{r^{2}} \zeta\right\},
\end{gathered}
$$




$$
\Delta \psi-\frac{1}{r} \frac{\partial \psi}{\partial r}=\mathbf{r} \zeta
$$

with boundary conditions for $\Gamma, \zeta$ and $\psi$ given by

$$
\begin{aligned}
& \Gamma\left(\frac{\mathrm{R}_{0}}{\mathrm{~L}}, z\right)=\left(\frac{\mathrm{R}_{0}}{\mathrm{~L}}\right)^{2}, \quad \psi\left(\frac{\mathrm{R}_{0}}{\mathrm{~L}}, z\right)=0, \quad \zeta\left(\frac{\mathrm{R}_{0}}{\mathrm{~L}}, z\right)=\frac{\mathrm{L}}{\mathrm{R}_{0}} \frac{\partial^{2} \psi}{\partial \mathrm{r}^{2}}\left(\frac{\mathrm{R}_{0}}{\mathrm{~L}}, z\right) . \\
& \text { for } 0 \leq z \leq 1 \text {, } \\
& \Gamma\left(\frac{\mathrm{R}_{\mathrm{I}}}{\mathrm{L}}, \mathrm{z}\right)=\left(\frac{\mathrm{R} I}{\mathrm{~L}}\right)^{2}, \quad \psi\left(\frac{\mathrm{R}_{\mathrm{L}}}{\mathrm{L}}, \mathrm{z}\right)=0, \quad \zeta\left(\frac{\mathrm{R}_{\mathrm{I}}}{\mathrm{L}}, \mathrm{z}\right)=\frac{\mathrm{L}}{\mathrm{R}_{\mathrm{I}}} \frac{\partial^{2} \psi}{\partial \mathrm{r}^{2}}\left(\frac{{ }_{\mathrm{R}} \mathrm{I}}{\mathrm{L}}, z\right), \\
& \text { for } 0 \leq z \leq 1 \text {, } \\
& \Gamma(\mathrm{r}, 0)=\mathrm{r}^{2}, \quad \psi(\mathrm{r}, 0)=0, \quad \zeta(\mathrm{r}, 0)=\frac{1}{\mathrm{r}} \frac{\partial^{2} \psi}{\partial z^{2}}(\mathrm{r}, 0), \\
& \text { for } \frac{R_{I}}{L} \leq r \leq \frac{R_{0}}{L}
\end{aligned}
$$

and

$$
\begin{gathered}
\Gamma(r, 1)=\psi(r, 1)=0, \quad \zeta(r, 1)=\frac{1}{r} \frac{\partial^{2} \psi}{\partial z^{2}}(r, 1) \\
\text { for } \frac{R_{I}}{L} \leq r \leq \frac{R_{0}}{L} .
\end{gathered}
$$

These latter boundary conditions are obtained from Equations (3.5) (3.8) with the aid of the definitions given in Equations (2.18), (2.24) and (2.30) and the transformation given in Equation (2.27). 
In the particular problem for which solutions were attempted,

$$
\begin{aligned}
& R_{0}=0.5 \mathrm{ft} . \\
& R_{I}=0.03125 \mathrm{ft} . \\
& L=1.0 \mathrm{ft} ., \\
& \nu=0.0364 \mathrm{ft}^{2} / \mathrm{hr} .
\end{aligned}
$$

and $\Omega$ is deterimined for any tangential Reynolds number Re from Equation (2.33). It should be noted that Kidd [7] defined the tangential Reynolds number by

$$
\mathrm{Re}_{K}=\frac{\mathrm{R}_{0}^{2} \Omega}{v}
$$

and it is clear from Equations $(3.16),(3.18)$ and $(3.19)$ that

$$
\operatorname{Re}_{K}=0.25 \operatorname{Re}
$$




\section{CHAPTER IV}

\section{THE CLASSICAL APPROACH}

For two problems very similar to the one posed in Chapter III, Pao [13] has made an attempt to solve both the steady-state equations (given by Equations (3.9) - (3.11)) and the transient equations (given by Equations (2.37) - (2.39)) numerically. In the problem of interest to Pao which is more closely related to the problem of Chapter III, the only differences are the absence of the center tube and incidental dimensions. Hereafter, all references to Pao [13] are to be understood as references to the solution of the steady-state version of this problem, unless otherwise noted. In his other problem only one end wall is rotating, i.e., the outside cylinder wall is held stationary with the other end wall. It is interesting to note that these two problems arose during an investigation of the dynamics of a cyclone or tornado over fixed ground.

The essential details of Pao's approach to the problem are as follows :

(1) Cover the rectangle of interest (Figure 4) with a network of parallel grid lines which are uniformly spaced by an amount $h$ in both the $r$ - and $z$-directions.

(2) Write finite difference equations (see [14]) which are analogous to Equations (3.9) - (3.11) and which give values for $\Gamma, \zeta$ and $\psi$ at each node (the intersection of two grid lines) of the grid in terms of the values of $\Gamma, \zeta$ and $\psi$ at neighboring nodes. 


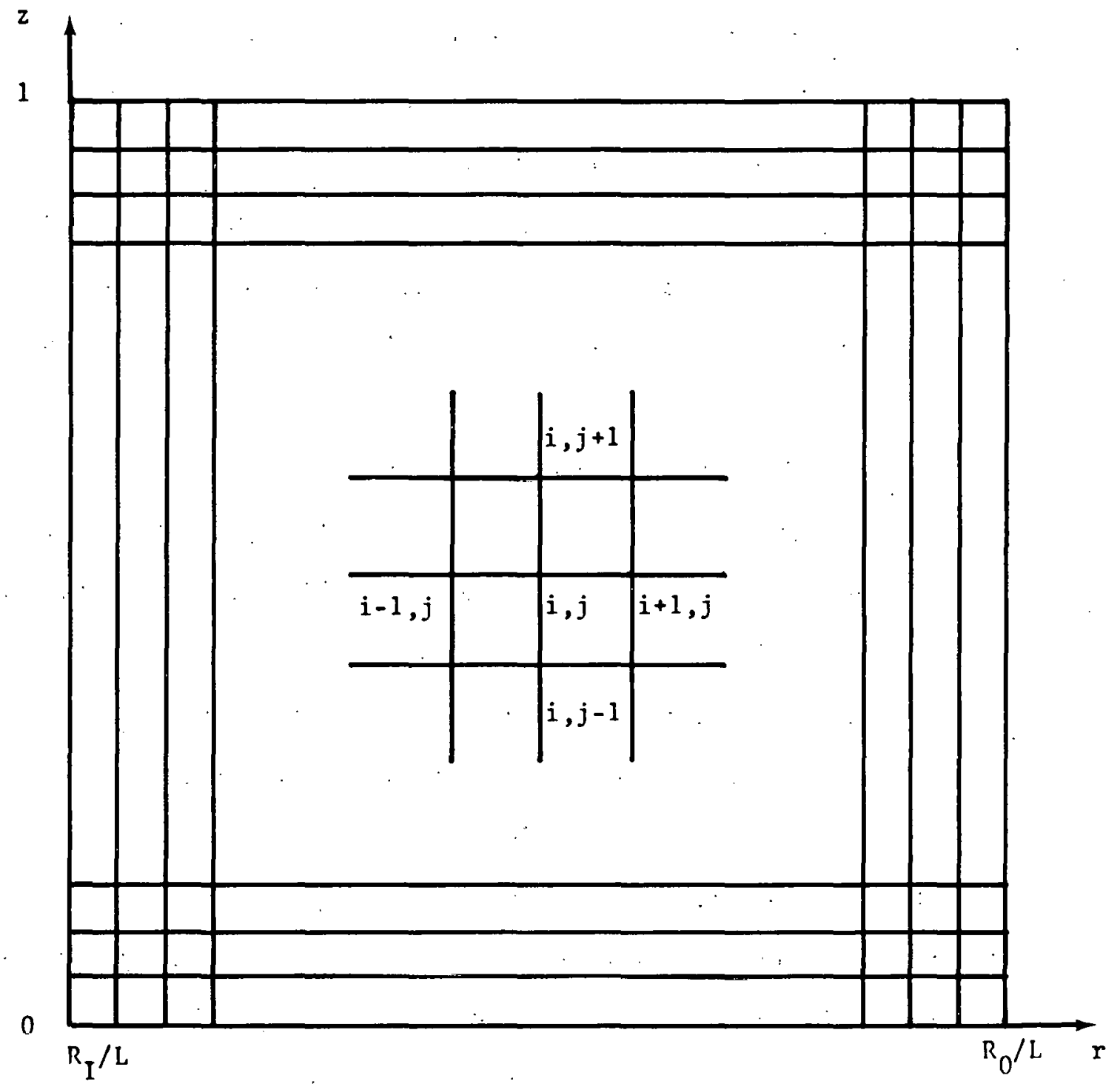

Figure 4. Structure of the Grid Network. 
(3) For a given value of the Reynolds number and for a given initial guess for $\Gamma, \zeta$ and $\psi$ at each of the nodes use a Gauss-Seidel procedure (see [14]) to solve for $\Gamma, \zeta$ and $\psi$ iteratively until some convergence criterion has been satisfied. Each iteration involves solving the finite difference equations for $\Gamma, \zeta$ and $\psi$ at each interior node, in turn. Because of the nature of the $\zeta$ boundary conditions given in Equations (3.12) - (3.15), a new set of boundary conditions for $\zeta$ must be determined at the end of each iteration.

(4) Once "convergence" has been attained, use finite difference analogues of Equations (2.18) and (2.31), in conjunction with the transformations of Equation (2.27), to obtain the radial, tangential and axial velocities at the nodal points.

(5) Use the values of $\Gamma, \zeta$ and $\psi$ for the case of the Reynolds number just concluded as an initial guess for the case of the next larger Reynolds number of interest.

We note that Pao defined the tangential Reynolds number as in Equation (2.33) and that increasing the Reynolds number in effect magnifies the importance of the nonlinear terms in Equations (3.9) and (3.10). Indeed, increasing the Reynolds number in these equations ultimately results in a numerical instability (i.e., divergence rather than convergence) which is directly analogous to the instability (i.e., turbulent flow) which ultimately results from increasing the Reynolds number (by increasing the angular velocity $\Omega$ of the outer cylinder) during an experiment. Since it 
is often possible to observe experimentally an upper limit on the Reynolds number for laminar flow, the goal of the mathematician is the development of finite difference representations of the partial differential equations and iterative procedures which also remain stable until the limiting Reynolds number is reached.

For any interior nodal point (designated by the indices $i$, in the r-direction, and $j$, in the $z$-direction) the finite difference equations analogous to Equations (3.9) - (3.11) can be written as

$$
\begin{aligned}
& \Gamma_{i, j}=\frac{1}{4}\left\{\Gamma_{i+1, j}+\Gamma_{i-1, j}+\Gamma_{i, j+1}+\Gamma_{i, j-1}\right\} \\
& -\frac{\operatorname{Re}}{16 r_{i}}\left\{\left[\psi_{i, j+1}-. \psi_{i, j-1}+\frac{2 h}{\operatorname{Re}}\right]\left[\Gamma_{i+1, j}-\Gamma_{i-1, j}\right]\right. \\
& \left.-\left[\psi_{i+1, j}-\psi_{i-1, j}\right]\left[\Gamma_{i, j+1}-\Gamma_{i, j-1}\right]\right\}, \\
& \zeta_{i, j}=\left\{\frac{1}{4}\left[\zeta_{i+1, j}+\zeta_{i-1, j}+\zeta_{i, j+1}+\zeta_{i, j-1}\right]\right. \\
& -\frac{\operatorname{Re}}{16 r_{i}}\left[\left(\psi_{i, j+1}-\psi_{i, j-1}-\frac{2 h}{\operatorname{Re}}\right)\left(\zeta_{i+1, j}-\zeta_{i-1, j}\right)\right. \\
& -\left(\psi_{i+1, j}-\psi_{i-1, j}\right)\left(\zeta_{i, j+1}-\zeta_{i, j-1}\right) \\
& \left.\left.-\frac{4 h \Gamma_{i, j}}{\left(r_{i}\right)^{2}}\left(\Gamma_{i, j+1}-\Gamma_{i, j-1}\right)\right]\right\} \div\left\{1+\left(\psi_{i, j-1}-\psi_{i, j+1}+\frac{2 h}{\operatorname{Re}}\right) \frac{h R e}{8\left(r_{i}\right)^{2}}\right\},
\end{aligned}
$$

and 


$$
\begin{aligned}
& \psi_{i, j}=\frac{1}{4}\left\{\psi_{i+1, j}+\psi_{i-1, j}+\psi_{i, j+1}+\psi_{i, j-1}\right\} \\
& -\frac{h}{8 r_{i}}\left\{2\left(r_{i}\right){ }^{2} \zeta_{i, j}+\psi_{i+1, j}-\psi_{i-1, j}\right\}
\end{aligned}
$$

when central difference approximations are used for the required derivatives.

Pao applied these finite difference equations to each nodal point of a grid which covered the unit square and had the mesh size $h=0.025$. The boundary conditions used are those given in Equations (3.12) (3.15) except that the boundary at $r=0$ is stationary, hence $\Gamma=\zeta=0$ at $r=0$. For this problem Pao was able to obtain solutions for $\operatorname{Re}<400$ and found that the computation diverged at $\mathrm{Re}=400$. He then suggested that $\mathrm{Re}=400$ is the upper limit for the steady-state approach, since he was able to obtain a solution for the analogous transient problem at $\operatorname{Re}=5000$. His calculations were carried out in single precision on an IBM 7040 digital computer.

Obviously, the success reported by Pao suggested that the classical stream function-vorticity approach might provide a reasonable degree of success in an attack upon the problem at hand. Indeed, there was justification for anticipating an extension of his results to higher Reynolds numbers. It has been noted that Pao used a Gauss-Seidel iteration procedure. For a number of linear partial differential equations it can be shown (see [15]) that the method of Successive Overrelaxation (S.O.R.) is far superior. In fact, this method has been used by Tejeira [12], among others, to accelerate convergence for some Reynolds numbers and to 
decelerate the numerical process in order to prevent divergence at other values of the Reynolds number in the solution of flow problems. In addition to the use of the S.O.R. iterative method, it was decided that a smaller mesh size (namely, $h=0.015625=1 / 64$ ) and double precision arithmetic would be used. in hopes that, while the accuracy of the results obtained by Pao could be enhanced, any instability resulting from roundoff errors could be overcome. Since the machine that was to be used is the IBM Model 360/75, these alterations are certainly within practical limits with regard to memory size and operating speed.

Convergence of the solutions for $\Gamma, \zeta$ and $\psi$ was considered to have occurred when

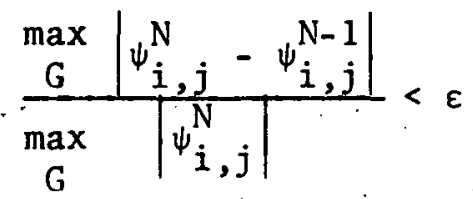

where $G$ is the set of the interior nodal points of the grid, the superscript $N$ denotes the current iteration, and usually $\varepsilon=0.001$, although occasionally $\varepsilon=0.0001$. It has been observed that $\psi$ is the most sensitive of the three variables, i.e., when the stream function distribution satisfies the inequality of Equation (4.4), the $\zeta$ and $\Gamma$ distributions satisfy analogous convergence criteria. Since one of the primary objectives of this effort was to obtain solutions for as large values of the tangential Reynolds number as possible, the primary interest in the solution at any particular Reynolds number was that it provide a sufficiently good initial guess for the solutions at the next larger Reynolds number. For this reason the stated value of $\varepsilon$ was chosen rather than a smaller value. 
For the first case $(\operatorname{Re}=1)$ it was assumed, as an initial guess, that the majority of the fluid was in solid-body rotation, i.e:,

$$
\mathbf{u}=\mathbf{w}=0, \quad \mathbf{v}=\mathbf{r} \Omega,
$$

and that as $z \rightarrow 1, v \rightarrow 0$ and $u=w=0$. The relaxation factor $w$ was found to vary inversely with the Reynolds number. For Re $=1$, the use of $\omega=1.5$ led to convergence of the solution we1l within 200 iterations and it was found that the iterative procedure could be successfully overrelaxed for Reynolds numbers as large as $\operatorname{Re}=364$, although the number of iterations required for convergence gradually increased to -600 iterations at $\operatorname{Re}=364$. Convergence of the solution at $\operatorname{Re}=400$ required 2600 iterations with $\dot{\omega}=1.0$. It should be noted that $\operatorname{Re}=400$ was the value of the Reynolds number for which Pao [13] observed divergence of the solution. Since the use of $\omega=1$ in the S.O.R. iterative procedure is equivalent to the use of the Gauss-Seidel procedure, the reasons for this "paradox" are not evident, for the importance of the accuracy of the initial guess, the grid size and the type of floating point arithmetic (single vs. double precision) to convergence have not been fully explored for nonlinear partial difference equations. However; during the convergence of the $\operatorname{Re}=400$ case it was observed that

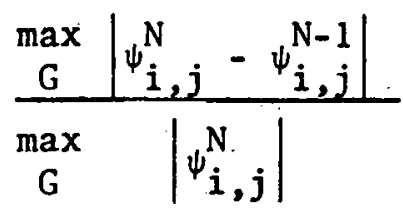

increased over several hundred iterations before decreasing to convergence. 
For $\operatorname{Re}>400$, underrelaxation $(\omega<1)$ has been found to be a requirement for numerical stability and ultimate convergence of the solution. This fact reiterates the "special" nature of this value of the tangential Reynolds number for this problem. To date solutions have been obtained for Reynolds numbers as high as 2000 , the value of the relaxation factor has decreased to $w-0.05$ and the number of iterations required for solution has been uniformly 600-800 iterations for $R e>400$. 


\section{CHAPTER $V$}

\section{OTHER APPROACHES}

While the numerical solution of the stream function-vorticity equations was the first approach to the solution of the problem of Chapter III that has provided a reasonable degree of success, there are other approaches which have provided little or no success, or which seem promising but have yet to be tried.

The first attempt at obtaining solutions for the problem of interest was made through a similarity transformation (mentioned in Chapter III), the basis for which is discussed by Rosenhead [16]. The form of the solution of the Navier-Stokes equations is assumed to be known, and then a suitable transformation is found which reduces the Navier-Stokes equations to a system of ordinary differential equations. Hopefully, these equations can then be integrated to obtain detailed velocity data. However, experimental verification of the results thus obtained is usually necessary since it is not always possible to obtain physically realistic velocity distributions. We consider the similarity transformation made by Kidd and Farris [17] which met with limited success. The classical stream function $\psi$ was defined such that

$$
\mathrm{u}=\frac{1}{\mathbf{r}} \frac{\partial \psi}{\partial z}, \quad w=-\frac{1}{r} \frac{\partial \psi}{\partial \mathbf{r}},
$$

and it was assumed that

$$
\psi=\psi\left(R_{0}, \Omega, v, r\right) f(n),
$$




$$
\begin{aligned}
& v=v\left(R_{0}, \Omega, v, r\right) g(n), \\
& P=P\left(p, R_{0}, \Omega, r\right) p(n),
\end{aligned}
$$

and

$$
\eta=n\left(R_{0}, \Omega, z, v, r\right)
$$

where $f$ is a dimensionless stream function, $g$ is a dimensionless angular velocity, $p$ is a dimensionless pressure, and $\eta$ is a dimensionless distance. By means of a dimensional analysis of Equations (3.2) - (3.4) for the problem of interest it is found that we must have

$$
\begin{aligned}
& \psi=\left(\operatorname{Re}_{K}\right)^{1 / 2} v r f(n), \\
& v=r \Omega g(n), \\
& P=\rho\left(\frac{R_{0}^{2} \Omega}{r}\right)^{2} p(n),
\end{aligned}
$$

and

$$
n=\left(\operatorname{Re}_{K}\right)^{1 / 2} \frac{z}{r}
$$

where $\operatorname{Re}_{K}$ is defined by Equation (3.20). Upon the substitution of Equations (5.6) - (5.9) into Equations (3.2) - (3.4), we obtain the system of ordinary differential equations 


$$
\begin{gathered}
\left.\left(1+\frac{\eta^{2}}{R e_{K}}\right) f^{\prime \prime}=-\left(f^{\prime}\right)^{2}-\left(f+\frac{3 n}{R e_{K}}\right) f^{\prime \prime}-g^{2}-n p^{\prime}-2 p\right) \\
\left(1+\frac{n^{2}}{R e_{K}}\right) g^{\prime}=-\left(f+\frac{3 n}{R e_{K}}\right) g^{\prime},
\end{gathered}
$$

and

$$
\left(1+\frac{n^{2}}{R e_{K}}\right) p^{\prime}=\frac{1}{R e_{K}}\left(1+\frac{n^{2}}{R e_{K}}\right) f^{\prime}+\frac{\eta f^{\prime}}{R e_{K}^{2}}-\frac{f}{R e_{K}^{2}}-\frac{f f^{\prime}}{R e_{K}}-\frac{2 n p}{R e_{K}}-\frac{n g^{2}}{R e_{K}},
$$

where the primes indicate differentiation with respect to the single variable $n$, e.g.,

$$
f^{\prime \prime} \equiv \frac{d^{3} f}{d n^{3}}
$$

For the idealized potential vortex of Kidd [7] the boundary conditions are as follows:

(1). The radial and tangential velocities ( $u$ and $v$, respectively) vanish at the stationary end wall.

(2). The stream function $\psi$ vanishes at the stationary end wall.

(3). The radial velocity $u$ vanishes far from the end wall.

(4). The tangential velocity approaches a potential vortex far from the end wall, i.e.,

$$
\mathbf{v}=\mathbf{r} \Omega
$$

In terms of the dimensionless quantities $f, g$ and $p$, these boundary conditions are 


$$
\begin{gathered}
f(0)=f^{\prime}(0)=g(0)=f^{\prime}(\infty)=0, \\
g(\infty)=1,
\end{gathered}
$$

and

$$
n p^{\prime}(\infty)+2 p(\infty)=-1
$$

With these boundary conditions, Equations (5.10) - (5.12) were numerically integrated to obtain values for $f, g$ and $p$ from which values for $u, v$, $w$ and $P$ may be obtained. Unfortunately, solutions could not be obtained for $\operatorname{Re}_{K}>4.75$.

As is indicated by Equations (3.5) - (3.8), very little is known about boundary conditions for the pressure P in Equations (3.1) - (3.4). To overcome that lack of knowledge one may differentiate Equation (3.2) with respect to $z$ and Equation (3.4) with respect to $r$ and subtract the results to obtain

$$
\begin{aligned}
& \left(\frac{\partial u}{\partial r}+\frac{\partial w}{\partial z}\right)\left(\frac{\partial u}{\partial z}-\frac{\partial w}{\partial r}\right)+u\left(\frac{\partial^{2} u}{\partial r^{\partial} z}-\frac{\partial^{2} w}{\partial r^{2}}\right)+w\left(\frac{\partial^{2} u}{\partial z^{2}}-\frac{\partial^{2} w}{\partial r^{\partial z}}\right)-2 \frac{v}{r} \frac{\partial v}{\partial z} \\
= & v\left\{\frac{\partial^{3} u}{\partial z^{3}}-\frac{\partial^{3} w}{\partial r^{3}}+\frac{\partial^{3} u}{\partial r^{2} \partial z}-\frac{\partial^{3} w}{\partial r \partial z^{2}}+\frac{1}{r}\left(\frac{\partial^{2} u}{\partial r \partial z}-\frac{\partial^{2} w}{\partial r^{2}}\right)+\frac{1}{r^{2}}\left(\frac{\partial w}{\partial r}-\frac{\partial u}{\partial z}\right)\right\}
\end{aligned}
$$

which, with Equations (3.1) and (3.3), provides a system of three partial differential equations, one of which is linear and first order, one of which is semi-linear and second order, and the other of which is semi-linear and third order. . An attempt was made by Farris, Lick and Dunphey [18] to solve Equations (3.1), (3.3) and (5.13), with the boundary conditions of Equations (3.5) - (3.8), using the S.O.R. 
iteration procedure on a grid with rectangular spacing, i.e., while the spacing of the grid lines was constant in the r-direction and in the zdirection, the spacing in the r-direction differed from that in the $z$ direction. This approach was numerically unstable, even when the procedure was severely under-relaxed, i.e., the relaxation factor $\omega$ was given values on the order of 0.01 , and when the finite difference analogues of the complicated derivatives in Equation (5.13) were altered. The reasons for this total numerical instability are not yet known, but several possible explanations exist. The finite difference analogues of the third order partial derivatives in Equation (5.13) may contribute inherent numerical instability, or perhaps the difference analogues of these and other derivatives should be forward or backward differences rather than central differences. The use of rectangular rather than square grid spacing may contribute sufficient divisions by "small" quantities so that numerical instability is the result. In this regard, note that in Equations (4.1) - (4.3) the grid size $h$ never appears in a denominator.

A most promising method which has not yet been tried for the problem of interest is the MAC method of Welch, Harlow, Shannon and Daly [19]. This approach differs from the majority of the literature treating the numerical solution of the Navier-Stokes equations in that it seeks to solve the equations directly for the velocity and pressure distributions rather than indirectly from solutions to the stream function-vorticity equations. Recall from Chapter II that the NavierStokes equations are partial differential equations which result from 
conserving mass and momentum in an incremental element of volume. The finite difference analogues of partial derivatives most often used are those which result from the truncation of Taylor series expansions of the unknown functions about nodal points of the grid, and the errors usually considered in the approximations thus obtained are the errors which result from this truncation process. A great deal of the success obtained by the authors of the MAC method can be attributed to the fact that the relation of the points at which the unknown functions are to be evaluated to the grid structure is such that the finite difference equations conserve both mass and momentum. One may imagine these finite difference equations as having been obtained from an application of conservation of mass and momentum to a finite volume element, as in Chapter II, thus omitting the intermediate step of writing the partial differential equations. For the moment we consider transient flow and write Equations $(2.20)-(2.22)$ as

$$
\begin{gathered}
\frac{\partial u}{\partial t}+u \frac{\partial u}{\partial r}=\frac{v^{2}}{r}+w \frac{\partial u}{\partial z}=-\frac{\partial \Phi}{\partial r}+v\left\{\frac{\partial}{\partial r}\left(\frac{1}{r} \frac{\partial}{\partial r}(r u)\right)+\frac{\partial^{2} u}{\partial z^{2}}\right\} \\
\frac{\partial v}{\partial t}+u \frac{\partial v}{\partial r}+\frac{u v}{r}+w \frac{\partial v}{\partial z}=v\left\{\frac{\partial}{\partial r}\left(\frac{1}{r} \frac{\partial}{\partial r}(r v)\right)+\frac{\partial^{2} v}{\partial z^{2}}\right\}
\end{gathered}
$$

and

$$
\frac{\partial w}{\partial t}+u \frac{\partial w}{\partial r}+w \frac{\partial w}{\partial z}=-\frac{\partial \Phi}{\partial z}+v\left\{\frac{1}{r} \frac{\partial}{\partial r}\left(r \frac{\partial w}{\partial r}\right)+\frac{\partial^{2} w}{\partial z^{2}}\right\}
$$

where 


$$
\Phi(r, z, t) \equiv \frac{1}{p} P(r, z, t)
$$

Equation (2.19) may be written as

$$
D(r, z, t)=\frac{1}{r} \frac{\partial}{\partial r}(r u)+\frac{\partial w}{\partial z}
$$

where the incompressibility condition is satisfied when the discrepancy $D \equiv 0$. The terms in Equations $(5.14)$ - (5.16) of which $\nu$ is the coefficient are respectively the $\ddot{r}-, \theta-$, and z-components of $\Delta \vec{v}$, where $\vec{v}$ is the velocity vector. The incompressibility condition can be written vectorially as

$$
\nabla \cdot \vec{v}=0, \ldots
$$

where $\nabla$ is the vector differential operator

$$
\nabla \equiv \frac{1}{\mathbf{r}} \frac{\partial}{\partial \mathbf{r}} \hat{\mathbf{i}}+\frac{\partial}{\partial z} \hat{\mathrm{k}}
$$

and $\hat{i}$ and $\hat{k}$ are unit vectors in the $r$ - and $z$-directions, respectively. We now use the vector identity

$$
\nabla \times \nabla \times \vec{v}=\nabla(\nabla \cdot \vec{v})-\Delta \vec{v}
$$

to rewrite Equations $(5.14)-(5.16)$ as

$$
\begin{aligned}
& \frac{\partial u}{\partial t}+u \frac{\partial u}{\partial r}-\frac{v^{2}}{r}+w \cdot \frac{\partial u}{\partial z}=-\frac{\partial \Phi}{\partial r}+v\left\{\frac{\partial}{\partial z}\left(\frac{\partial u}{\partial z}-\frac{\partial w}{\partial r}\right)\right\} \\
& \frac{\partial v}{\partial t}+u \frac{\partial v}{\partial r}+\frac{u v}{r}+w \frac{\partial v}{\partial z}=v\left\{\frac{\partial}{\partial r}\left(\frac{1}{r} \frac{\partial}{\partial r}(r v)\right)+\frac{\partial^{2} v}{\partial z^{2}}\right\}
\end{aligned}
$$


and

$$
\frac{\partial w}{\partial t}+u \frac{\partial w}{\partial r}+w \frac{\partial w}{\partial z}=-\frac{\partial \Phi}{\partial z}+v\left\{\frac{1}{r} \frac{\partial}{\partial r}\left(r\left(\frac{\partial w}{\partial r}-\frac{\partial u}{\partial z}\right)\right)\right\}
$$

Note that the nonlinear terms in the left member of Equation (5.19) may be written as.

$$
u \frac{\partial u}{\partial r}+w \frac{\partial u}{\partial z}=\frac{1}{r} \frac{\partial}{\partial r}\left(\dot{r u} u^{2}+\frac{\partial}{\partial z}(u w)\right.
$$

with the aid of the incompressibility condition. Similar expressions may be obtained for the nonlinear terms in the left members of Equations (5.20) and (5.21), and Equations (5.19) - (5.21) may be written as

$$
\begin{gathered}
\frac{\partial u}{\partial t}+\frac{1}{r} \frac{\partial}{\partial r}\left(r u^{2}\right)+\frac{\partial}{\partial z}(u w)-\frac{v^{2}}{r}=-\frac{\partial \Phi}{\partial r}+v\left\{\frac{\partial}{\partial z}\left(\frac{\partial u}{\partial z}-\frac{\partial w}{\partial r}\right)\right\} \\
\frac{\partial v}{\partial t}+\frac{1}{r} \frac{\partial}{\partial r}(r u v)+\frac{\partial}{\partial z}(v w)+\frac{u v}{r}=v\left\{\frac{\partial}{\partial r}\left(\frac{1}{r} \frac{\partial}{\partial r}(r v)\right)+\frac{\partial^{2} v}{\partial z^{2}}\right\}
\end{gathered}
$$

and

$$
\left.\frac{\partial w}{\partial t}+\frac{1}{r} \frac{\partial}{\partial r}(r u w)+\frac{\partial}{\partial z}\left(w^{2}\right)=-\frac{\partial \Phi}{\partial z}+v\left\{\frac{1}{r} \frac{\partial}{\partial r}\left(\frac{\partial w}{\partial r}-\frac{\partial u}{\partial z}\right)\right)\right\} .
$$

We now obtain an expression for the time rate of change in the discrepancy D from Equation (5.18), given by

$$
\frac{\partial D}{\partial t}=\frac{1}{r} \frac{\partial}{\partial r}\left(r \frac{\partial u}{\partial t}\right)+\frac{\partial}{\partial z}\left(\frac{\partial w}{\partial t}\right)
$$

into which we substitute the expressions for $\partial u / \partial t$ and $\partial w / \partial t$, given by Equations $(5.23)$ and $(5.25)$, and obtain 


$$
\begin{aligned}
\frac{1}{r} \frac{\partial}{\partial r}\left(r \frac{\partial \Phi}{\partial r}\right)+\frac{\partial^{2} \Phi}{\partial z^{2}} & =-\frac{1}{r} \frac{\partial^{2}}{\partial r^{2}}\left(r u^{2}\right)+\frac{1}{r} \frac{\partial}{\partial r}\left(v^{2}\right)-\frac{\partial^{2}}{\partial z^{2}}\left(w^{2}\right) \\
& -\frac{2}{r} \cdot \frac{\partial}{\partial r}\left(r \frac{\partial}{\partial \dot{z}}(u w)\right)-\frac{\partial D}{\partial t}
\end{aligned}
$$

which may be recognized as a cylindrical coordinate Poisson's equation for $\Phi$. The procedure of the MAC method involves solving Equation (5.27) iteratively for the pressure term $\Phi$ at any given time step and substituting the values thus obtained in Equations (5.23) and (5.25) to determine the radial and axial velocities at the next time step. Finally, the angular velocity at the next time step may be determined from Equation $(5,24)$

A network of grid lines with equal spacing is constructed in the region of interest forming a set of cells each of which is bounded by two parallel grid lines in the r-direction and by two parallel grid lines in the $z$-direction, as in Figure 5 . Let the indices $i$ (r-direction) and $j$ (z-direction) denote the center of any cell in the set, then the relation of the variables $u, v, w, \Phi$ and $D$ to the $i, j-c e l l$ is that shown in Figure 5. The finite difference representations of Equations $(5.23)$, $(5.24),(5.25)$ and $(5.27)$ are written as 


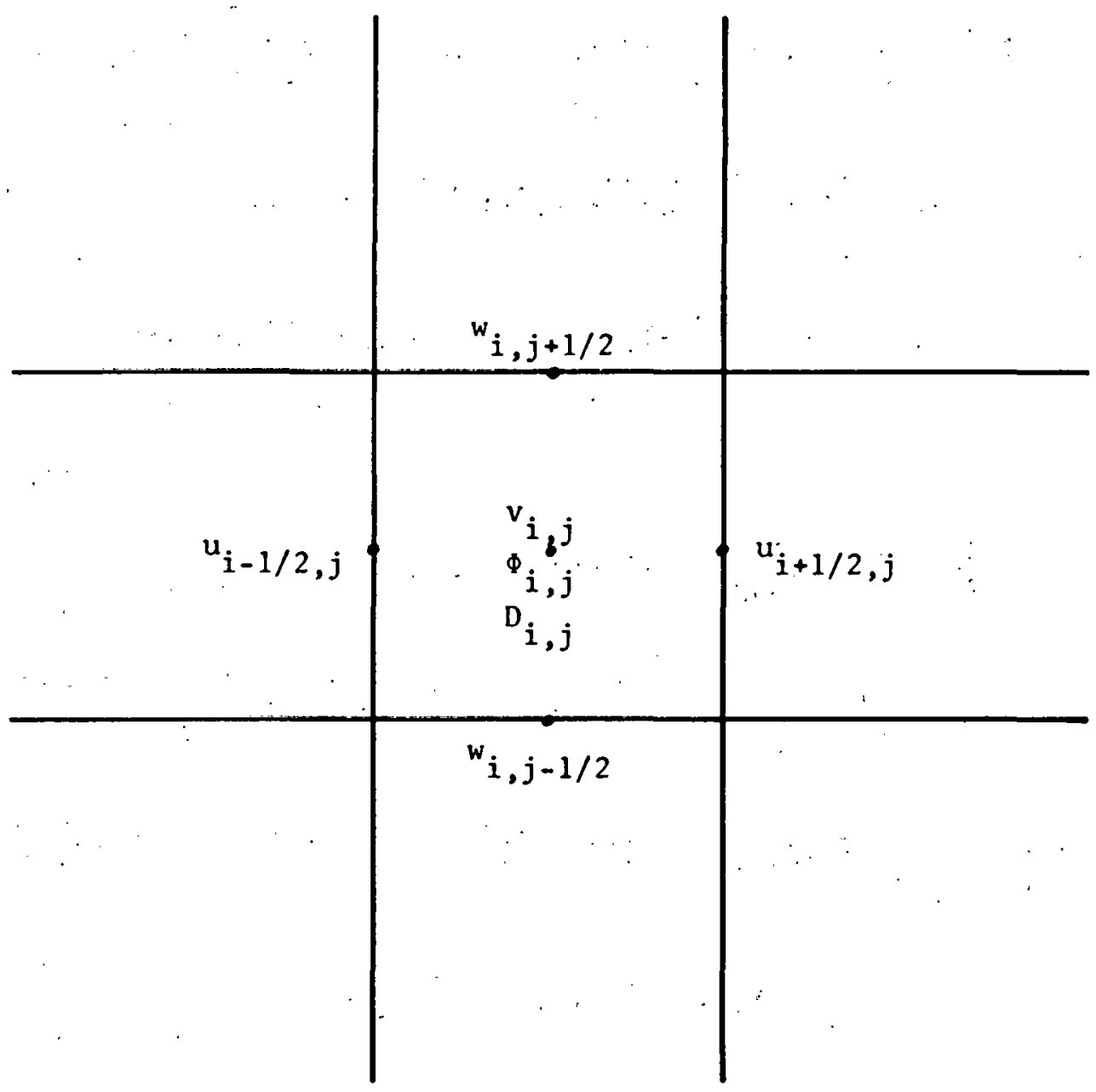

Figure 5. Relation of Field Variables to Grid Structure 


$$
\begin{aligned}
& \frac{1}{\delta t}\left\{u_{i+1 / 2, j}^{N+1}-u_{i+1 / 2, j}^{N}\right\}=\frac{1}{r_{i+1 / 2}}\left\{\left(r u^{2}\right)_{i, j}-\left(r u^{2}\right)_{i+1, j}\right\} \\
& +\frac{1}{h}\left\{(u w)_{i+1 / 2, j-1 / 2}-(u w)_{i+1 / 2, j+1 / 2}\right\}+\frac{1}{r_{i+1 / 2}}\left(v^{2}\right)_{i+1 / 2, j} \\
& +\frac{1}{h}\left\{\Phi_{i, j} \vdots \Phi_{i+1, j}\right\}+\frac{\nu}{h^{2}}\left\{u_{i+1 / 2, j+1}+u_{i+1 / 2, j-1}-2 u_{i+1 / 2, j}\right. \\
& \left.-w_{i+1, j+1 / 2}+w_{i+1, j-1 / 2}+w_{i, j+1 / 2}-w_{i, j-1 / 2}\right\}, \\
& \left.\frac{1}{\delta t}\left\{v_{i, j}^{N+1}-v_{i, j}^{N}\right\}=\frac{1}{r_{i} h^{(r u v)}}{ }_{i-1 / 2, j}-(\text { ruv })_{i+1 / 2, j}\right\} \\
& +\frac{1}{h}\left\{(v w)_{i, j-1 / 2}-(v w)_{i, j+1 / 2}\right\}=\frac{1}{r_{i}}(u v)_{i, j} \\
& +\frac{v}{h^{2}}\left[\frac{1}{r_{i+1 / 2}}\left[(r v)_{i+1, j}-(r v)_{i, j}\right]-\frac{1}{r_{i-1 / 2}}\left[(r v)_{i, j}-(r v)_{i-1, j}\right]\right. \\
& \left.+v_{i, j+1}+v_{i, j-1}-2 v_{i, j}\right\}
\end{aligned}
$$




$$
\begin{aligned}
& \frac{1}{\delta t}\left\{w_{i, j+1 / 2}^{N+1}-w_{i, j+1 / 2}^{N}\right\}=\frac{1}{r_{i}}\left\{(\text { ruw })_{i-1 / 2, j+1 / 2}-(\text { ruw })_{i+1 / 2, j+1 / 2}\right\} \\
& \quad+\frac{1}{h}\left\{\left(w^{2}\right)_{i, j}-\left(w^{2}\right)_{i, j+1}\right\}+\frac{1}{h}\left\{\Phi_{i, j}-\Phi_{i, j+1}\right\} \\
& +\frac{v}{r_{i} h^{2}}\left\{r_{i+1 / 2}\left(w_{i+1, j+1 / 2}-w_{i, j+1 / 2}-u_{i+1 / 2, j+1}+u_{i+1 / 2, j}\right)^{(5,30)}\right. \\
& \left.\quad-r_{i-1 / 2}\left(w_{i, j+1 / 2}-w_{i-1, j+1 / 2}-u_{i-1 / 2, j+1}+u_{i-1 / 2, j}\right)\right\}
\end{aligned}
$$

and

$$
\begin{aligned}
& \left.\frac{1}{r_{i} h^{2}}\left\{r_{i+1 / 2}\left(\Phi_{i+1, j}-\Phi_{i, j}\right)-r_{i-1 / 2}{ }^{\left(\Phi_{i, j}\right.}-\Phi_{i-1, j}\right)\right\} \\
& +\frac{1}{h^{2}}\left\{\Phi_{i, j+1}+\Phi_{i, j-1}-2 \Phi_{i, j}\right\}=\frac{1}{r_{i} h^{\{}}\left\{\left(r u^{2}\right)_{i, j}-\left(r u^{2}\right)_{i+1, j}-\left(r u^{2}\right)_{i-1, j}\right\} \\
& +\frac{1}{2 r_{i} h^{2}}\left(\left(v^{2}\right)_{i+1, j}-\left(v^{2}\right)_{i-1, j}\right\}+\frac{1}{h^{2}}\left\{2\left(w^{2}\right)_{i, j}-\left(w^{2}\right)_{i, j+1}-\left(w^{2}\right)_{i, j-1}\right\}
\end{aligned}
$$

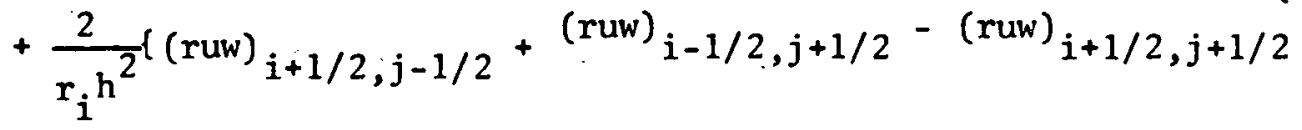

$$
\begin{aligned}
& \left.-(\text { ruw })_{i-1 / 2, j-1 / 2}\right\}-\frac{1}{\delta t}\left(D_{i, j}^{N+1}-D_{i, j}^{N}\right) \text {, }
\end{aligned}
$$

where $h$ is the grid size for the spatial coordinates, $\delta t$ is the time increment, and the superscripts $N$ and $N+1$ denote the times $N \delta t$ and $(N+1) \delta t$, respectively. A superscript of $N$ is to be understood in quantities for which a superscript is not written. Values of the variables 
required at points of the grid at which they are not defined are computed as average values, e.g.,

$$
(u w)_{i+1 / 2, j-1 / 2}=\frac{1}{4}\left(u_{i+1 / 2, j}+u_{i+1 / 2, j-1}\right)\left(w_{i+1, j-1 / 2}+w_{i-1, j-1 / 2}\right) \text {. }
$$

Suitable finite difference equations for the steady-state problem may be obtained by setting the time differences in Equations (5.28) - (5.31) to zero and solving Equations $(5.28)-(5.30)$ for the variables $u_{i+1 / 2, j}$, $v_{i, j}$ and $w_{i, j+1 / 2}$, respectively. After the provision of boundary conditions for the pressure term $\Phi$, a possible iteration procedure for the steady-state problem would be as follows:

(1). Solve Equation (5.31) for the $\phi_{i, j}$ in an iterative fashion (perhaps S.O.R.) until some convergence criterion has been satisfied.

(2). Use the values for the $\dot{\phi}_{i, j}$ just calculated to determine new values for $u, v$ and $w$.

(3): Use the new values for $u, v$ and $w$ to determine a new $\phi-$ distribution and continue the process until $u, v$ and $w$ have also satisfied some convergence criteria:

The MAC method was designed to handle time-dependent, incompressible, viscous fluid flow problems which involve free surface boundaries (such as air or another fluid) as well as solid boundaries (such as the problem at hand). That the MAC method is capable of successfully treating a wide variety of problems is attested to by the problems discussed in [19], and certainly the problem of present interest to this author would fail to use all the features available in the method. 
In two recent papers, Chorin $[20,21]$ proposes a numerical method involving an implicit alternating direction scheme (see [14]) for solving either the transient or the steady-state Navier-Stokes equations directly for the velocity and pressure distributions. He states that the method is equally applicable to problems in two and three space dimensions, although his preliminary results indicate that there are important differences between the behavior of the solutions in two and three space dimensions. 
THIS PAGE

\section{WAS INTENTIONALLY LEFT BLANK}




\section{LIST OF REFERENCES}

1. Bird, R. B., W. E. Stewart, and E. N. Light foot, Transport Phenomena, John Wiley and Sons, New York, 1960.

2. Kerrebrock, J. L., and J. J. Keyes, Jr., A Preliminary Experimental Study of Vortex Tubes for Gas Phase Fission Heating, USAEC Report, ORNL-2660, Oak Ridge National Laboratory, Oak Ridge, Tennessee (1959).

3. Kerrebrock, J. L., and R. V. Meghreblian, Vortex Containment for the Gaseous-Fission Rocket, J. Aerospace Sci. 28 (1961), 710-724.

4. Keyes, J. J., Jr., Experimental Study of Flow and Separation in Vortex Tubes with Application to Gaseous Fission Heating, ARS J. 31 (1961), 1204-1210.

5. Kenda11, J. M., Jr., Experimental Study of a Compressible Viscous Vortex, Technical Report No. 32-290, California Institute of Technology, Pasadena, California (1962).

6. Rosenzweig, M. L., W. S. Lewellen, and D. H. Ross, Confined Vortex Flows with Boundary-Layer Interaction, Report No. ATN-64(9227)-2, Aerospace Corporation, El Segundo, California (1964).

7. Kidd, G. J., Jr., Confined Vortex Flow Near a Stationary Disk, Theory and Experiment (Thesis), USAEC Report, ORNL-TM-1387, Oak Ridge National Laboratory, Oak Ridge, Tennessee (1966).

8. Fromm, J. E., A Method for Computing Nonsteady, Incompressible, Viscous Fluid Flows, USAEC Report, LA-2910, Los Alamos Scientific Laboratory, Los Alamos, New Mexico (1963).

9. Pearson, C. E., A Computational Method for Time-Dependent Two Dimensional Incompressible Viscous Flow Problems, Report SRRC-RR64-17, Sperry Rand Research Center, Sudbury, Massachusetts (1964).

10. Pearson, C. E., A Computational Method for Viscous Flow Problems, $J$ Fluid Mech. 21 (1965), 611-622.

11. Pearson, C. E., Numerical Solutions for the Time Dependent Viscous Flow Between Two Rotating Coaxial Disks, J Fluid Mech. 21 (1965), 623-633.

12. Tejeira, E. J., Numerical and Experimental Investigation of a TwoDimensional Laminar Flow with Non-Regular Boundaries, Report EM 66-8-1, Department of Engineering Mechanics, University of Tennessee, Knoxville, Tennessee (1966). 
13. Pao, Hsien-Ping, A Numerical Computation of a Confined Vortex, Report No. 67-024, Department of Space Science and Applied Physics, The Catholic University of America, Washington, D. C. (1967).

14. Forsythe, G. E., and W. R. Wasow, Finite Difference Methods for Partial Differential Equations, John Wiley and Sons, New York, 1960.

15. Varga, R. S., Matrix Iterative Analysis, Prentice-Hall, Englewood C1iffs, New Jersey, 1962.

16. Rosenhead, L., Laminar Boundary Layers, Clarendon Press, Oxford, 1963.

17. Kidd, G. J., Jr., and G. J. Farris, "Potential Vortex Flow Adjacent to a Stationary Surface," submitted for publication to ASME (1967).

18. Farris, G. J., D. W. Lick, and J. E. Dunphey, (Private Communication).

19. Welch, J. E., F. H. Harlow, J. P. Shannon, and B, J. Daly, The MAC Method, A Computing Technique for Solving Viscous, Incompressible; Transient Fluid-Flow Problems Involving Free Surfaces, USAEC Report, LA-3425, Los Alamos Scientific Laboratory, Los Alamos, New Mexico (1966).

20. Chorin, A. J., A Numerical Method for Solving Incompressible Viscous Flow Problems, submitted for publication to J. Comp. Phys. (1967).

21. Chorin, A. J., The Numerical Solution of the Navier-Stokes Equations for an Incompressible Fluid, to appear in Bull. A.M.S. 73 (1967). 